\title{
DISCRETE LIMIT AND MONOTONICITY PROPERTIES OF THE FLOQUET EIGENVALUE IN AN AGE STRUCTURED CELL DIVISION CYCLE MODEL
}

\author{
StÉPHANe GaUBerT \\ INRIA Saclay Île-de-France and CMAP, École Polytechnique \\ Postal address: CMAP, École Polytechnique, 91128 Palaiseau cedex, France \\ Thomas Lepoutre \\ INRIA Rhône Alpes (team DRACULA) \\ Batiment CEI-1, 66 Boulevard NIELS BOHR, 69603 Villeurbanne cedex, France \\ Université de Lyon CNRS UMR 5208 \\ Université Lyon 1 Institut Camille Jordan \\ 43 blvd. du 11 novembre 1918 F-69622 Villeurbanne cedex France
}

\begin{abstract}
We consider a cell population described by an age-structured partial differential equation with time periodic coefficients. We assume that division only occurs after a minimal age (majority) and within certain time intervals. We study the asymptotic behavior of the dominant Floquet eigenvalue, or Perron-Frobenius eigenvalue, representing the growth rate, as a function of the majority age, when the division rate tends to infinity (divisions become instantaneous). We show that the dominant Floquet eigenvalue converges to a staircase function with an infinite number of steps, determined by a discrete dynamical system. As an intermediate result, we give a structural condition which guarantees that the dominant Floquet eigenvalue is a nondecreasing function of the division rate. We also give a counter example showing that the latter monotonicity property does not hold in general.
\end{abstract}

\section{InTRODUCTION}

1.1. Age structured model of the cell division cycle. Among all the population models, age-structured population models are perhaps the simplest and

oldest ones taking into account the variability between individuals. Age structured partial differential equations have been introduced in epidemiology through the famous Von Foerster - Mac Kendrick model [12] (where age stands for age in the disease). In its simplest version, it describes the dynamics of a population represented by a density $n(t, x)$ of individuals of age between $x$ and $x+d x$ through age-dependent birth and death rate:

2010 Mathematics Subject Classification. 35B40, 35Q92, 35P15.

Key words and phrases. cell cycle, circadian rhythms, structured PDEs, Perron-Frobenius theory, Floquet eigenvalue, delay differential equations. 


$$
\left\{\begin{array}{l}
\frac{\partial n}{\partial t}+\frac{\partial n}{\partial x}+d(x) n(t, x)=0 \\
n(t, 0)=\int_{0}^{\infty} B(x) n(t, x) d x \\
n(0, x)=n^{0}(x) \quad \text { given }
\end{array}\right.
$$

A special case of this model can be used to represent cell division, taking $d(x)=K(x)$ and $B(x)=2 K(x)$. More complex models, in terms of structure, representing the cell division cycle (linear or not, size or cyclin structured) have been developped and studied in $[16,10,21]$. In this paper, we study an extension of the age-structured division equation, in which the coefficients are periodic functions of time. There are several motivations to introduce time dependence in (1). The most classical one is the representation of seasonality (see [2] for instance). Concerning cell division, our main motivation comes from cancer therapy such as resonance therapy [8] or chronotherapy [13]. The latter is based on the use of circadian rhythms and motivates especially the introduction of time periodic coefficients (see $[7,11]$ for instance). We also refer to $[9,14,19]$ for work on renewal models with periodic coefficients.

In our case, the periodic division equation reads

$$
\left\{\begin{array}{l}
\frac{\partial n}{\partial t}+\frac{\partial n}{\partial x}+K(t, x) n(t, x)=0 \\
n(t, 0)=2 \int_{0}^{\infty} K(t, x) n(t, x) d x \\
n(0, x)=n^{0}(x) \quad \text { given. }
\end{array}\right.
$$

where $K$ satisfies $K(t+T, \cdot)=K(t, \cdot)$ for some $T>0$.

For equations (1),(2), the population grows exponentially with time (see [18] for instance) and the growth rate is measured by the so called Malthus parameter $\lambda$ $\left(n(t, x) \sim \rho e^{\lambda t} N(t, x), N\right.$ being given by (3)). Particularly, in (2), the Malthus parameter $\lambda_{F}$ (F for Floquet) can be generally described by means of a Floquet eigenvalue problem.

$$
\left\{\begin{array}{l}
\frac{\partial N}{\partial t}+\frac{\partial N}{\partial x}+\left(\lambda_{F}+K(t, x)\right) N(t, x)=0 \\
n(t, 0)=2 \int_{0}^{\infty} K(t, x) N(t, x) d x \\
N(t+T, x)=N(t, x), N>0
\end{array}\right.
$$

together with the dual eigenproblem

$$
\left\{\begin{array}{l}
-\frac{\partial \phi}{\partial t}-\frac{\partial \phi}{\partial x}+\left(\lambda_{F}+K(t, x)\right) \phi(t, x)=2 K(t, x) \phi(t, 0) \\
\phi(t+T, x)=\phi(t, x), \phi>0 .
\end{array}\right.
$$

For uniqueness issues, these equations are usually completed with the following normalization:

$$
\frac{1}{T} \int_{0}^{T} \int_{0}^{\infty} N d x d t=\frac{1}{T} \int_{0}^{T} \int_{0}^{\infty} N(t, x) \phi(t, x) d x d t=1 .
$$


Actually, the integral $\int_{0}^{\infty} N(t, x) \phi(t, x) d x$ does not depend on $t$. Various properties of the Floquet eigenvalue have been studied in $[2,3,4,5,6]$ with in mind the comparison with time independent systems (in which $K(t, x)$ is replaced by suitable time averages). Here, we are interested in properties of the eigenvalue (and more generally of the dynamics) that are intrinsically related to the fact that the coefficients are time-dependent.

We consider in Section 3 a division rate of the form

$$
K(t, x)=\kappa \psi(t) B(x) \mathbb{1}_{[a,+\infty[}(x) .
$$

Here, $\kappa>0$ is a scaling parameter and the function $\psi(t) \geqslant 0$ expresses the dependence of the division rate as a function of time. We denote by $\mathbb{1}$ the indicator function of a set. The term $B(x) \mathbb{1}_{[a,+\infty[}(x)$ indicates that the cells only divide when $x \geqslant a$, i.e., when they are older than a "majority age" $a$. The function $B(x)$ expresses a general modulation of the division rate as a function of the age.

The function $B$ will be required to be bounded, positive, of infinite integral,

$$
B \in L^{\infty}\left(\mathbb{R}^{+}\right), \quad B>0, \quad \int_{\mathbb{R}^{+}} B=+\infty
$$

This is needed for the existence theory of the Floquet eigenvalue (see the appendix).

1.2. Main results. Our goal is to derive the asymptotics of the growth rate when the division becomes instantaneous, meaning that the scaling parameter $\kappa$ in (6) tends to $\infty$. The growth rate is represented by the Floquet eigenvalue, $\lambda_{F}$, which is the unique scalar $\lambda$ solution of (3),(4). Hence, we shall investigate the asymptotic behavior of $\lambda_{F}$ as a function of $\kappa$.

To this end, we first study the monotonicity of the Floquet eigenvalue with respect to the division coefficient $K(t, x)$. Since every division replaces one old cell by two new ones, it is natural to think that the growth rate is a nondecreasing function of $K(t, x)$. Our first result is an explicit counter example, showing that this apparently intuitive property is not valid due to the time and age dependence of the division rate.

Proposition 1. There exists configurations such that

$$
\forall(t, x), \quad 0 \leqslant K^{1}(t, x) \leqslant K^{2}(t, x),
$$

and the corresponding Floquet eigenvalues satisfy

$$
\lambda_{F}^{1}>\lambda_{F}^{2} .
$$

However, the following theorem identifies a subclass of transition rates for which the Floquet eigenvalue is indeed a monotone function of the transition rate.

Theorem 1.1 (Comparison principle). If $K^{1}$ satisfies

$$
v \mapsto \int_{v}^{t} K^{1}(s, s-v) d s \quad \text { is nondecreasing for any } t,
$$

then for all $K^{2} \geqslant 0$,

$$
\begin{aligned}
& \left(\forall(t, x) \quad K^{2}(t, x) \geqslant K^{1}(t, x)\right) \quad \Rightarrow \quad \lambda_{F}^{2} \geqslant \lambda_{F}^{1}, \\
& \left(\forall(t, x) \quad K^{2}(t, x) \leqslant K^{1}(t, x)\right) \quad \Rightarrow \quad \lambda_{F}^{2} \leqslant \lambda_{F}^{1} .
\end{aligned}
$$


The proof relies on a disaggregation idea of independent interest. We first show that the density $n(t, x)$ can be written as $\sum_{i \geqslant 0} 2^{i} n_{i}(t, x)$, where every $n_{i}(t, x)$ can be interpreted as the density of an individual of the $i$ th generation, and the densities $\left(n_{i}\right)_{i \geqslant 0}$ satisfy an infinite triangular system of transport equations coupled by integral terms. Then, for all $j \geqslant 0$, we show that the term $S_{j}(t)=\sum_{i \geqslant j} \int_{0}^{\infty} n_{i}(t, x) d x$ is a nondecreasing function of $K \in\left\{K^{1}, K^{2}\right\}$, and derive from this fact the comparison of Floquet eigenvalues. We note that the latter fact has a probabilistic interpretation: it means precisely that the generation of a single individual is a nondecreasing function of $K \in\left\{K^{1}, K^{2}\right\}$ with respect to the stochastic (majorization) order.

When $\inf \psi>0$, the intuition predicts that the growth rate goes to the limit $\log 2 / a$ as $\kappa$ tends to infinity, because every cell will divide shortly after reaching age $a$ when the parameter $\kappa$ is large. Our next result confirms that this is the case.

Proposition 2. Suppose that $\psi$ is a bounded T-periodic function with inf $\psi>0$, and that $B$ is a positive bounded function satisfying (7).

Then, for all $\kappa>0$,

$$
0 \leqslant \lambda_{F}(\kappa) \leqslant \frac{\log (2)}{a}
$$

and

$$
\lim _{\kappa \rightarrow \infty} \lambda_{F}(\kappa)=\frac{\log (2)}{a} .
$$

When $\psi$ vanishes, meaning that the division of cells is blocked at certain times of the day, we shall see that complex synchronization phenomena appear. We shall assume that $\psi$ has a square wave shape, i.e., that $\psi$ is a $T$-periodic function such that, for some $0<\tau<T$,

$$
\psi(t)=\left\{\begin{array}{ll}
1 & \text { for } t \in[0, \tau) \\
0 & \text { for } t \in[\tau, T)
\end{array} .\right.
$$

The following is the main result of this paper. We denote by $\lceil x\rceil$ the smallest integer which is not less than a real number $x$.

Theorem 1.2 (Discrete limit). Suppose that the division rate is given by $K(t, x)=$ $\kappa \psi(t) \mathbb{1}_{[a, \infty)}(x)$, where $\psi$ is given by (10). Denote $W_{\tau}=[\tau, T]+\mathbb{N} T$ and let $N_{a}$ be the integer defined by

$$
N_{a} a=\min W_{\tau} \cap \mathbb{N} a .
$$

Then, the limit of the Floquet eigenvalue $\lambda^{\kappa}(a)$ as $\kappa \rightarrow+\infty$ is given by

$$
\lambda^{\infty}(a)=\frac{N_{a}}{\left\lceil N_{a} a / T\right\rceil T} \log 2 .
$$

Moreover, we have the estimate

$$
\lambda^{\infty}(a, \tau) \geqslant \lambda^{\kappa}(a, \tau) \geqslant \lambda^{\infty}(a)\left(1-e^{-\kappa r(a, \tau)}\right),
$$

where the function $r(a, \tau)$ (the convergence rate) satisfies

$$
r(a, \tau) \geqslant \min \left(\frac{a_{r}-a}{2}, \tau\right), \quad a_{r}=\sup \left\{a^{\prime}, \lambda^{\infty}\left(a^{\prime}\right)=\lambda^{\infty}(a)\right\},
$$

and $r$ is positive outside a closed countable set. 
The function $a \mapsto \lambda^{\infty}(a)$ is a staircase with a countable number of steps (see Figure 1 below and Figure 8 and Figure 9 in Section 3.5). The quantity $a_{r}-a$ which controls the rate represents the distance to the right end of the step containing $a$ (on Figure 1 for instance, we can observe that the speed of convergence depends on this distance).

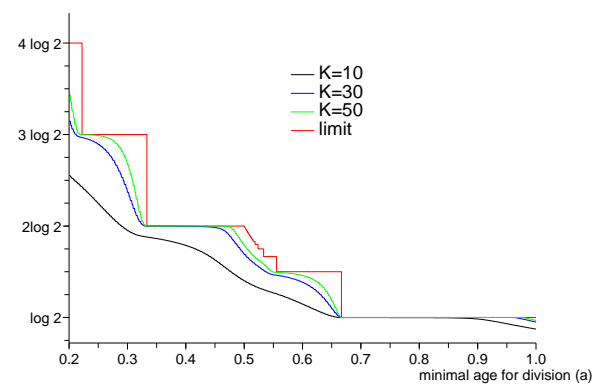

Figure 1. Convergence for $\tau=2 / 3$ and $T=1$.

There is a special case in which the limit of the Floquet eigenvalue can be rewritten in terms of integer parts. If $\tau / T<1 / 2$, and $0<a<T$, then, we have

$$
\lambda^{\infty}(a)=\left\lceil\frac{\tau}{a}\right\rceil \log 2 .
$$

We shall derive intuitively Formula (11) in Section 3.2.1, by introducing a discrete dynamical system, equipped with a multiplicative functional, the asymptotic geometric mean of which yields $\lambda^{\infty}(a)$. The existence of such a simple asymptotic formula reflects the fact that the Perron-Frobenius operator describing the evolution of the population degenerates as $\kappa \rightarrow \infty$. The rate $r(a)$ appears naturally in the proof of convergence. This proof relies on Theorem 1.1 (the comparison principle), on the generational model already used in the proof of this theorem, and on explicit bounds of the density, for a special initial condition leading to a trajectory "close" to that of the discrete dynamical system.

The convergence rate in Theorem 1.2 is reminiscent of large-deviation or Laplace asymptotics. However, this theorem differs in its essence of already known asymptotics results in Perron-Frobenius theory with such large deviations flavor, including zero-temperature asymptotics of Ising type models or Frenkel-Kantorova models $[1,20]$. In these problems, the limit of the Perron eigenvalue, in a log-scale, turns out to be the ergodic constant of a controlled deterministic dynamical system, whereas in the present case, the dynamical system which determines the limit involves no control.

When $\psi$ vanishes, we may consider $\psi^{\varepsilon}(t):=\psi(t)+\varepsilon$, with $\varepsilon>0$, and define the growth rate $\lambda(\epsilon, \kappa)$ with an obvious notation. A comparison of the previous theorems shows that the limits in $\kappa$ and $\varepsilon$ do not commute

$$
\frac{\log (2)}{a}=\lim _{\varepsilon \rightarrow 0} \lim _{\kappa \rightarrow \infty} \lambda(\epsilon, \kappa) \neq \lim _{\kappa \rightarrow \infty} \lim _{\varepsilon \rightarrow 0} \lambda(\epsilon, \kappa)=\lambda^{\infty}(a) .
$$




\section{Monotonicity With Respect to The Division RATE}

We first focus on the question of the monotonicity of the Floquet eigenvalue with respect to the division rate.

2.1. A counter example in which the growth is not a monotone function of the division rate. We next show that increasing the division rate may decrease the growth rate of the population. The counter example is based on the following exactly solvable model. We assume that the period is $T=1$, and fix $\alpha \in(0,1)$. It will be convenient to write

$$
I_{1}=[0, \alpha)+\mathbb{Z}, \quad I_{2}=[\alpha, 1)+\mathbb{Z},
$$

and

$$
\chi_{j}(t):=\mathbb{1}_{I_{j}}(t), \quad j=1,2, \quad \text { so that } \chi_{1}+\chi_{2}=1 .
$$

We shall assume that the division rate is of the form

$$
K(t, x)=\chi_{1}(t-x) K_{1}(t)+\chi_{2}(t-x) K_{2}(t) .
$$

Integrating along a characteristic, we get

$$
n(t, x)=n(t-x, 0) \exp \left(-\int_{0}^{x} K(t-x+y, y) d y\right)
$$

and so

$$
n(t, x)=n(t-x, 0) \exp \left(-\int_{0}^{x} K_{j}(t-x+y) d y\right), \quad \text { if } t-x \in I_{j} .
$$

In other words, when an individual is born at time $t \in I_{j}$, then its next division will occur with a rate $K_{j}(t)$. This model has an intuitive interpretation. We may assume for instance that $I_{1}$ represents the days, whereas $I_{2}$ represents the nights. If being born during the night or during the day influences the fertility (division rate) of the individuals, we arrive at this model. We may then consider that there are actually two subpopulations $n_{j}(t, x), j=1,2$, corresponding respectively to individuals born during the day or during the night. We have

$$
n_{j}(t, x)=n(t, x) \chi_{j}(t-x), \quad j=1,2
$$

They satisfy the equations:

$$
\left\{\begin{array}{l}
\partial_{t} n_{j}+\partial_{x} n_{j}+K_{j}(t) n_{j}=0, \quad j=1,2 \\
n_{j}(t, 0)=2 \chi_{j}(t) \int_{0}^{\infty}\left(K_{1}(t) n_{1}(t, x)+K_{2}(t) n_{2}(t, x)\right) d x \quad j=1,2 .
\end{array}\right.
$$

Denoting by

$$
P_{j}(t)=\int_{0}^{\infty} n_{j}(t, x) d x
$$

the total population of type $j$ at time $t$, we get that the vector $P(t):=\left(P_{1}(t), P_{2}(t)\right)^{\top}$ satisfies the ODE:

$$
\frac{d}{d t} P(t)=M(t) P(t)
$$

where

$$
M(t):=\left(\begin{array}{cc}
\left(2 \chi_{1}(t)-1\right) K_{1}(t) & 2 \chi_{1}(t) K_{2}(t) \\
2 \chi_{2}(t) K_{2}(t) & \left(2 \chi_{2}(t)-1\right) K_{2}(t)
\end{array}\right)
$$


We now assume that the functions $K_{1}(t)$ and $K_{2}(t)$ are constant during the day and during the night, meaning that

$$
K_{1}(t)=a_{1} \chi_{1}(t)+b_{1} \chi_{2}(t), \quad K_{2}(t)=a_{2} \chi_{1}(t)+b_{2} \chi_{2}(t),
$$

where the coefficients $a_{i}, b_{i}$ are constant. With this form of the coefficients, we obtain

$$
M(t)=\chi_{1}(t) M_{a}+\chi_{2}(t) M_{b}
$$

where

$$
M_{a}:=\left(\begin{array}{cc}
a_{1} & 2 a_{2} \\
0 & -a_{2}
\end{array}\right), \quad M_{b}:=\left(\begin{array}{cc}
-b_{1} & 0 \\
2 b_{1} & b_{2}
\end{array}\right) .
$$

An important point here, still with the above interpretation is the following: $a_{2}$ and $b_{1}$ could be considered in some sense as transition coefficients between populations $n_{1}$ and $n_{2}$.

Since the linear dynamics $M(t)$ switches between the constant dynamics $M_{a}$ and $M_{b}$, which are successively exercised during the intervals $[0, \alpha)$ and $[\alpha, 1)$, we get that

$$
P(1)=\exp \left((1-\alpha) M_{b}\right) \exp \left(\alpha M_{a}\right) P(0) .
$$

Denoting by $\rho$ the Perron root (spectral radius) of a nonnegative matrix, we arrive at the following expression for the Floquet eigenvalue

$$
\lambda_{F}=\log \rho\left(\exp \left((1-\alpha) M_{b}\right) \exp \left(\alpha M_{a}\right)\right) .
$$

Let us now consider the situation in which $a_{2}=b_{2}=0$, meaning that an individual born during the night is sterile (division will never occur). Then, the second columns of the matrices $M_{a}$ and $M_{b}$ vanish, and so

$$
\exp \left((1-\alpha) M_{b}\right) \exp \left(\alpha M_{a}\right)=\left(\begin{array}{cc}
\exp \left(\alpha a_{1}-(1-\alpha) b_{1}\right) & 0 \\
\star & 1
\end{array}\right)
$$

(the value of the off diagonal entry, denoted by $\star$, is irrelevant). Since the spectral radius of a nonnegative triangular matrix is the maximum of its of its diagonal entries, we get

$$
\lambda_{F}=\max \left(\alpha a_{1}-(1-\alpha) b_{1}, 0\right) .
$$

Hence, $\lambda_{F}$ is an increasing function of the division rate $a_{1}$, but a decreasing function of the division rate $b_{1}$. This establishes Proposition 1 .

It should be noted that the preceding counter-example subsists when $a_{2}$ and $b_{2}$ are sufficiently small, because the spectral radius is a continuous function of the parameters. This is confirmed by Figure 2, in which the Floquet eigenvalue is plotted as a function of $b_{1}$ and $b_{2}$ for $a_{1}=10$ and $a_{2}=0.1$.

Remark 1. This counter example can be understood intuitively in Figure 3. The values of the division rate $K(t, x)$ are represented (Left, the values are constant on each cell). Consider now a chain of descendants of the same individual (i.e., this individual, one of his children, one of the children of this children, etc.). This can be represented by a Markov process $X_{t}$ with state space in $\mathbb{R}_{+}$(the age), which jumps from age $x$ to age 0 with rate $K(t, x)$. Each time the path hits the axis $x=0$ corresponds to a division. If the path hits $x=0$ during the night, since $b_{2}=0$, the corresponding individual is sterile (no division will ever occur, which is reflected that the path is now an unending line of slope 1 in the $(t, x)$ plane). The production of sterile individuals occurs when the process jumps from a cell labeled 


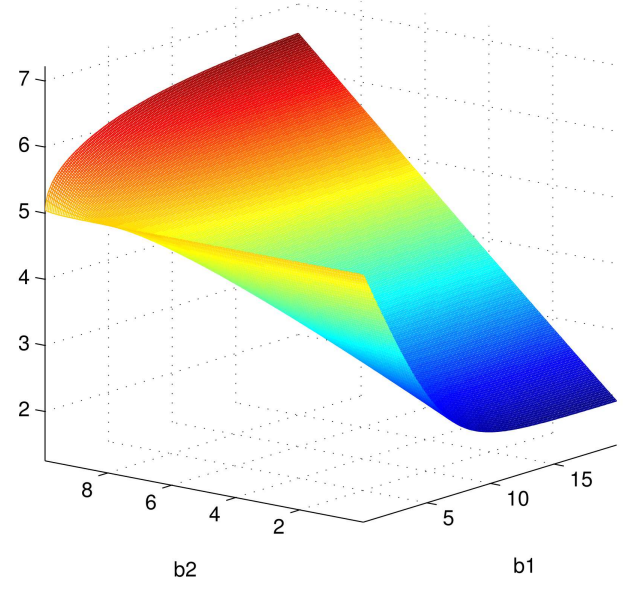

Figure 2. The counter example. The Floquet eigenvalue as a function of the division rates $b_{1}$ and $b_{2}$, when $a_{1}=10$ and $a_{2}=0.1$. When $b_{2}$ is small, increasing $b_{1}$ (and so, increasing the division rate $K(t, x))$ decreases the growth rate.

" $b_{1}$ " to the $x$ axis, and this occurs precisely with rate $b_{1}$, which explains intuitively why the growth rate, $\lambda_{F}$, is a decreasing function of $b_{1}$.
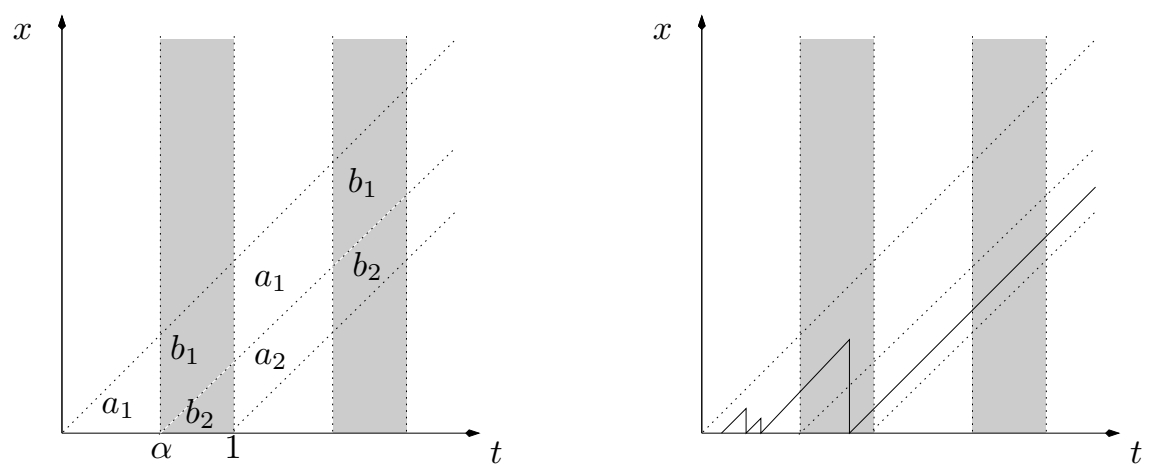

Figure 3. The counter example explained. The values of $K(t, x)$ (left). The night is shown in grey. A typical path (right, broken line). The child born during the night is sterile (final segment of the path), and the division coefficient $b_{1}$ determines the creation of this child.

Remark 2. The system does not enter in the framework considered in Appendix for the existence of the Floquet eigenvalue. Indeed, the preceding study merely determines the growth rate of the "aggregated" population $P(t)$, which is determined as the Perron eigenvalue of a matrix. However, the growth of $n(t, x)$ is readily derived from the one of $P(t)$. To see this, it is convenient to introduce the 
adjoint positive Floquet eigenvector of $M(t)$, whose existence and uniqueness are guaranteed by Perron-Frobenius theory (as soon as the coefficients $a_{1}, a_{2}, b_{1}, b_{2}$ are positive):

$$
-\frac{d}{d t}\left(\begin{array}{ll}
\phi_{1} & \phi_{2}
\end{array}\right)+\lambda\left(\begin{array}{ll}
\phi_{1} & \phi_{2}
\end{array}\right)+\left(\begin{array}{ll}
\phi_{1} & \phi_{2}
\end{array}\right) M(t)=0 .
$$

We have

$$
\frac{d}{d t}(\phi(t), P(t))=\lambda(\phi(t), P(t))
$$

which can be read as, denoting $\phi(t, x)=\phi_{1}(t) \chi_{1}(t-x)+\phi_{2}(t) \chi_{2}(t-x)$,

$$
\frac{d}{d t} \int_{0}^{\infty} n(t, x) \phi(t, x) d x=\lambda \int_{0}^{\infty} n(t, x) \phi(t, x) d x
$$

It is straightforward to check that $\phi_{1}$ and $\phi_{2}$ are positive (still assuming that the coefficients $a_{1}, a_{2}, b_{1}, b_{2}$ are positive). Hence, there exists $M \geqslant m>0$ such that $m \leqslant \phi(t, x) \leqslant M$, and therefore,

$$
m e^{\lambda t} \int n^{0} d x \leqslant \int n(t, x) d x \leqslant M e^{\lambda t} \int n^{0} d x .
$$

This shows that the Floquet eigenvalue $\lambda$ computed from the aggregated dynamical system does determine the behavior of the solutions.

2.2. A sufficient condition for monotonicity. The preceding counter example is due to a "birth day penalty" (the date of birth of the individual influences critically its division rate). We shall avoid such a pathological behavior by making the following assumption:

$$
(t, v) \mapsto \int_{v}^{t} K(s, s-v) d s \text { is nonincreasing in } v .
$$

This condition is fulfilled when the division rate $K(t, x) \geqslant 0$ depends only on one of the two variables $t$ and $x$. It is also fulfilled if for any $t, K(t, \cdot)$ is a nondecreasing function (and particularly, in the case of separated variables $K(t, x)=\psi(t) B(x)$ when $B$ is nondecreasing).

This is illustrated in Figure 4. The condition requires the integral of $K$ over the characteristic shown on the figure to be nonincreasing in $v$. Note that this has a probabilist interpretation in terms of the Markov process $X_{t}$ introduced in the preceding section. Indeed,

$$
\exp \left(-\int_{v}^{t} K(s, s-v) d s\right)
$$

represents the survival probability at time $t$ of an individual born at time $v$, i.e., the probability, conditional to $X_{v}=0$, that the process makes no jump to point 0 before time $t$. The condition requires this probability to be a nondecreasing function of $v$, in other words, that by delaying the birth, the survival probability at a given time cannot decrease.

Theorem 2.1. Provided $K^{1}$ or $K^{2}$ satisfies (15) and $K^{1} \geqslant K^{2}$, then, for any $n^{0} \in L^{1}\left(\mathbb{R}_{+}\right)$, if we denote by $n^{i}$ the solution of $(2)$ with $K=K^{i}$, we have, for any $t \geqslant 0$,

$$
\int_{0}^{\infty} n^{1}(t, x) d x \geqslant \int_{0}^{\infty} n^{2}(t, x) d x .
$$




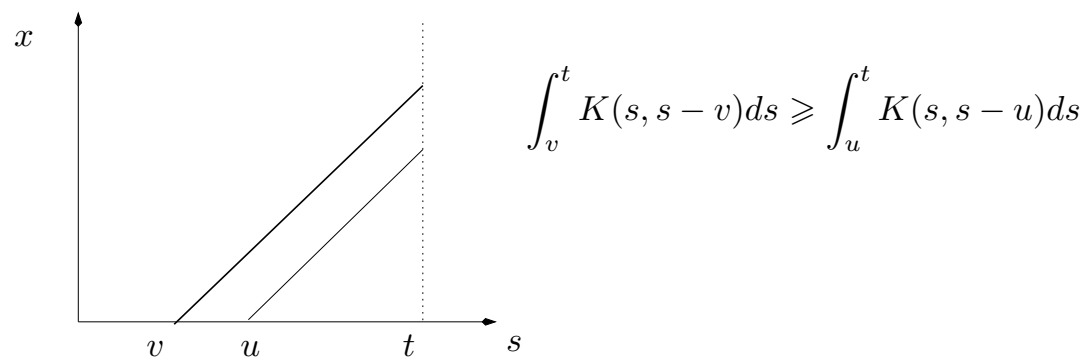

FIgURE 4. The sufficient monotonicity condition: the survival probability at time $t$ (the exponential of the opposite of the integral) increases with the birth time $v$.

To establish this result, we introduce the following system of PDEs:

$$
\left\{\begin{array}{l}
\partial_{t} n_{i}+\partial_{x} n_{i}+K(t, x) n_{i}=0, \quad i \geqslant 0 \\
n_{0}(t, 0)=0 \\
n_{i}(t, 0)=\int_{0}^{\infty} K(t, x) n_{i-1}(t, x) d x, \quad i \geqslant 0 \\
n_{0}(t=0, x)=n^{0}(x), \quad n_{i}(t=0, x)=0, \quad \text { for } i \geqslant 1 .
\end{array}\right.
$$

We easily check that $n=\sum_{i \geqslant 0} 2^{i} n_{i}$ is the solution to (2). Intuitively, the term $2^{i} n_{i}$ represents the number of individuals of the "generation" $i$, and so, we shall refer to this model as the "generational model" in the sequel. We denote by $\left(n_{i}^{1}\right)_{i \geqslant 0}$, (resp. $\left.\left(n_{i}^{2}\right)_{i \geqslant 0}\right)$ the solution to (16) with $K=K^{1}$ (resp. $\left.K=K^{2}\right)$. We also introduce:

$$
S_{j}(t):=\sum_{i \geqslant j} \int_{0}^{\infty} n_{i}(t, x) d x, \quad \text { for } j \geqslant 0 .
$$

A short computation leads to

$$
\int_{0}^{\infty} n(t, x) d x=S_{0}(t)+\sum_{j \geqslant 1} 2^{j-1} S_{j}(t) .
$$

Lemma 2.2. We have:

$$
\begin{aligned}
& S_{0}(t)=S_{0}(0)=\int_{0}^{\infty} n^{0}(x) d x, \\
& S_{1}(t)=\int_{0}^{\infty} n^{0}(x)\left(1-\exp \left(-\int_{0}^{t} K(s, x+s) d s\right)\right) d x,
\end{aligned}
$$

and for $j \geqslant 1$,

$$
S_{j}(t)=\int_{s=0}^{t} n_{j-1}(s, 0)\left(1-\exp \left(-\int_{0}^{t-s} K(s+y, y) d y\right)\right) d s .
$$

Proof. It is straightforward to show that for every $j \geqslant 0$,

$$
\frac{d}{d t} S_{j}(t)=n_{j}(t, 0)
$$


and so

$$
S_{j}(t)=\int_{0}^{t} n_{j}(s, 0) d s+S_{j}(0) .
$$

This leads immediately to the first statement, since for all $s>0, n_{0}(s, 0)=0$.

To establish the next statements, we shall use the characteristics:

$$
\begin{aligned}
& n_{0}(t, x)=0, \quad \text { if } x \leqslant t \\
& n_{0}(t, x)=n^{0}(x-t) \exp \left(-\int_{0}^{t} K(s, x-t+s) d s\right), \quad \text { if } x \geqslant t,
\end{aligned}
$$

and for $i \geqslant 1$,

$$
\begin{aligned}
& n_{i}(t, x)=0, \quad \text { if } t \leqslant x \\
& n_{i}(t, x)=n_{i}(t-x, 0) \exp \left(-\int_{0}^{x} K(t-x+y, y) d y\right), \quad \text { if } x \leqslant t .
\end{aligned}
$$

The lack of symmetry between the cases $i \geqslant 1$ and $i=0$ is due to the boundary conditions: $n_{0}(0, x)=n^{0}(x)$ is known, whereas for $i \geqslant 0, n_{i}(t, 0)$ can be inductively assumed to be known, using $n_{i-1}$.

Since $n_{1}(0, x)=0$, we have $S_{1}(0)=0$. Using (20), we get

$$
\begin{aligned}
S_{1}(t) & =\int_{s=0}^{t} n_{1}(s, 0) d s=\int_{s=0}^{t} \int_{x=0}^{\infty} K(s, x) n_{0}(s, x) d x d s \\
& =\int_{s=0}^{t} \int_{x=s}^{\infty} K(s, x) n^{0}(x-s) \exp \left(-\int_{0}^{s} K(u, x-s+u) d u\right) d x d s \\
& =\int_{s=0}^{t} \int_{y=0}^{\infty} K(s, y+s) n^{0}(y) \exp \left(-\int_{0}^{s} K(u, y+u) d u\right) d y d s
\end{aligned}
$$

and, interchanging the order of integration,

$$
\begin{aligned}
S_{1}(t) & =\int_{y=0}^{\infty} n^{0}(y) \int_{s=0}^{t} K(s, y+s) \exp \left(-\int_{0}^{s} K(u, y+u) d u\right) d s d y \\
& =\int_{0}^{\infty} n^{0}(y)\left(1-\exp \left(-\int_{0}^{t} K(s, y+s) d s\right)\right) d y
\end{aligned}
$$

which is the second statement.

Assume now that $j \geqslant 2$. Arguing as above, but using this time (22) instead of (21), we get

$$
\begin{aligned}
S_{j}(t) & =\int_{s=0}^{t} \int_{x=0}^{\infty} K(s, x) n_{j-1}(s, x) d x d s \\
& =\int_{s=0}^{t} \int_{x=0}^{s} K(s, x) n_{j-1}(s-x, 0) \exp \left(-\int_{0}^{x} K(s-x+y, y) d y\right) d x d s \\
& =\int_{x=0}^{t} \int_{s=x}^{t} K(s, x) n_{j-1}(s-x, 0) \exp \left(-\int_{0}^{x} K(s-x+y, y) d y\right) d s d x \\
& =\int_{x=0}^{t} \int_{u=0}^{t-x} K(u+x, x) n_{j-1}(u, 0) \exp \left(-\int_{0}^{x} K(u+y, y) d y\right) d u d x \\
& =\int_{u=0}^{t} n_{j-1}(u, 0) \int_{x=0}^{t-u} K(u+x, x) \exp \left(-\int_{0}^{x} K(u+y, y) d y\right) d x d u,
\end{aligned}
$$

which leads to the third statement. 
Theorem 2.1 will be obtained as an immediate consequence of the following lemma.

Lemma 2.3. Suppose $K^{1}$ satisfies (15), then

$$
\begin{array}{ll}
K^{2} \geqslant K^{1} \Longrightarrow S_{j}^{2}(t) \geqslant S_{j}^{1}(t), \quad \forall t, j \\
K^{2} \leqslant K^{1} \Longrightarrow S_{j}^{2}(t) \leqslant S_{j}^{1}(t), \quad \forall t, j .
\end{array}
$$

Proof. The proof is performed by induction on $j$. We shall only consider the case in which $K^{2} \geqslant K^{1}$, the other case being similar.

For $j=0,1$, the conclusion follows readily from the two first statements in Lemma 2.2. Assume now that $j \geqslant 2$. We set

$$
Q^{k}(t, v):=1-\exp \left(-\int_{0}^{t-v} K^{k}(v+y, y) d y\right)
$$

Using (19), we get

$$
\begin{aligned}
& S_{j}^{1}(t)-S_{j}^{2}(t)=\int_{0}^{t} n_{j-1}^{1}(v, 0) Q^{1}(t, v) d v-\int_{0}^{t} n_{j-1}^{2}(v, 0) Q^{2}(t, v) d v \\
& =\int_{0}^{t}\left(n_{j-1}^{1}(v, 0)-n_{j-1}^{2}(v, 0)\right) Q^{1}(t, v) d v+\int_{0}^{t} n_{j-1}^{2}(v, 0)\left(Q^{1}(t, v)-Q^{2}(t, v)\right) d v \\
& =\left[\left(S_{j-1}^{1}(v)-S_{j-1}^{2}(v)\right) Q^{1}(t, v)\right]_{0}^{t}-\int_{0}^{t}\left(S_{j-1}^{1}(v)-S_{j-1}^{2}(v)\right) \frac{d}{d v} Q^{1}(t, v) \\
& \quad+\int_{0}^{t} n_{j-1}^{2}(v, 0)\left(Q^{1}(t, v)-Q^{2}(t, v)\right) d v \\
& =-\int_{0}^{t}\left(S_{j-1}^{1}(v)-S_{j-1}^{2}(v)\right) \frac{d}{d v} Q^{1}(t, v)+\int_{0}^{t} n_{j-1}^{2}(v, 0)\left(Q^{1}(t, v)-Q^{2}(t, v)\right) d v
\end{aligned}
$$

The condition (15) ensures the nonpositivity of $\frac{d}{d v} Q^{1}(t, v)$. Moreover, $K^{2} \geqslant K^{1}$ readily implies $Q^{2} \geqslant Q^{1}$. Using the induction hypothesis, we deduce that $S_{j}^{2} \geqslant$ $S_{j}^{1}$.

Remark 3. Lemma 2.3 has an interpretation in terms of stochastic order or majorization [15]. Recall that for $\mathbb{R}$-valued random variables $Z^{1}, Z^{2}$, the stochastic order $\geqslant_{s t}$ is such that $Z^{2} \geqslant{ }_{s t} Z^{1}$ holds if and only if $\mathbb{P}\left(Z^{2} \geqslant t\right) \geqslant \mathbb{P}\left(Z^{1} \geqslant t\right)$ for all $t \in \mathbb{R}$. Now, let $X(t)$ denote the Markov process representing the age of a single individual, defined in Remark 1, and let $Y(t)$ denotes its number of jumps at time $t$, i.e., the "generation" of an individual. Then, $S_{j}(t)=\sum_{i \geqslant j} \int_{0}^{\infty} n_{i}(x, t) d t=\mathbb{P}(Y(t) \geqslant j)$, and so, denoting by $Y^{1}(t), Y^{2}(t)$ the two processes obtained in this way from $K^{1}, K^{2}$, we see that the properties of Lemma 2.3 are equivalent to

$$
\begin{array}{ll}
K^{2} \geqslant K^{1} \Longrightarrow Y^{2}(t) \geqslant_{s t} Y^{1}(t), \quad \forall t \\
K^{2} \leqslant K^{1} \Longrightarrow Y^{2}(t) \leqslant_{s t} Y^{1}(t), \quad \forall t .
\end{array}
$$

\section{Asymptotics of the Growth Rate}

We now assume that the division rate is of the form

$$
K(t, x)=\kappa \psi(t) B(x) \mathbb{1}_{[a,+\infty[}(x)
$$

an determine the limit of the Floquet eigenvalue of System (2) as the division rate tends to infinity, i.e., as the scaling parameter $\kappa$ tends to infinity. 
3.1. When both $\psi$ and $B$ are positive: proof of Theorem 2. The Floquet eigenvalue $\lambda_{F}^{\kappa}$ satisfies

$$
\left\{\begin{array}{l}
\frac{\partial}{\partial t} N^{\kappa}(t, x)+\frac{\partial}{\partial x} N^{\kappa}(t, x)+\left[\lambda_{F}^{\kappa}+\kappa \psi(t) B(x) \mathbb{1}_{[a,+\infty[}(x)\right] N^{\kappa}(t, x)=0 \\
N^{\kappa}(t, 0)=2 \kappa \psi(t) \int_{a}^{\infty} B(x) N^{\kappa}(t, x) d x \\
N^{\kappa}>0, T \text {-periodic. }
\end{array}\right.
$$

The existence of the Floquet eigenvector $N^{\kappa}$ is derived in the appendix as a consequence of the Krein-Rutman theorem. In particular, the function $B$ is required to be positive and of infinite integral. We normalize the Floquet eigenvector by requiring that

$$
\int_{0}^{T} \int_{0}^{\infty} N^{\kappa}(t, x) d x d t=1
$$

To establish Theorem 2, we shall think of the Floquet eigenvalue $\lambda_{F}^{\kappa}=\lambda_{F}^{\kappa}(\psi)$ as a function of the time modulation $\psi$. Since the division rate is a nondecreasing function of $\psi$, using the comparison principle (Theorem 1.1), we get

$$
\lambda_{F}^{\kappa}(\underline{\psi}) \leqslant \lambda_{F}^{\kappa}(\psi) \leqslant \lambda_{F}^{\kappa}(\bar{\psi}),
$$

where $\psi$ and $\bar{\psi}$ denote the constant functions equal to the minimum or maximum of $\psi$, respectively. When the function $\psi(t)$ is equal to a constant $\alpha$, the Floquet eigenvector can be chosen to be independent of $t$, so that $N^{\kappa}(t, x)=N^{\kappa}(x)$. Then, the Floquet eigenvalue $\lambda_{F}^{\kappa}(\alpha)$ is actually an ordinary eigenvalue. Indeed, it is the only scalar $\mu$ solution of the system

$$
\left\{\begin{array}{l}
\frac{d}{d x} N^{\kappa}(x)+\left[\mu+\kappa \alpha B(x) \mathbb{1}_{[a,+\infty[}(x)\right] N^{\kappa}(x)=0, \\
N^{\kappa}(0)=2 \kappa \alpha \int_{a}^{\infty} B(x) N^{\kappa}(x) d x \\
N^{\kappa}>0
\end{array}\right.
$$

Integrating the latter differential equation, we get

$$
N^{\kappa}(x)=N^{\kappa}(0) \exp \left(-\mu x-\kappa \alpha \int_{a}^{x} B(y) d y\right) .
$$

Using the boundary condition, and eliminating $N^{\kappa}(0)$, we determine $\mu$ via the following implicit equation:

$$
2 \int_{a}^{\infty} \kappa \alpha B(x) \exp \left(-\mu x-\kappa \alpha \int_{a}^{x} B(y) d y\right) d x=1
$$

Since $\mu=\lambda_{F}^{\kappa}(\alpha) \geqslant 0$ (in fact, $\mu>0$, see Theorem A.1 in the appendix), we have

$$
1 \leqslant 2 \exp (-\mu a) \int_{a}^{\infty} \kappa \alpha B(x) \exp \left(-\kappa \alpha \int_{a}^{x} B(y) d y\right) d x=2 \exp (-\mu a)
$$

and, taking $\alpha:=\bar{\psi}$, we get

$$
\lambda_{F}^{\kappa}(\psi) \leqslant \lambda_{F}^{\kappa}(\bar{\psi}) \leqslant \frac{\log 2}{a} .
$$

To bound the Floquet eigenvalue from below, we perform an integration by parts, so that:

$$
1=2 \exp (-\mu a)-\mu \int_{a}^{\infty} \exp \left(-\mu x-\kappa \alpha \int_{a}^{x} B(y) d y\right) d x .
$$


Take now $\alpha:=\underline{\psi}$. Using the fact that $0<\lambda_{F}^{\kappa}(\underline{\psi})$ and a dominated convergence argument, we deduce that

$\int_{a}^{\infty} \exp \left(-\lambda_{F}^{\kappa}(\underline{\psi}) x-\kappa \underline{\psi} \int_{a}^{x} B(y) d y\right) d x \leqslant \int_{a}^{\infty} \exp \left(-\kappa \underline{\psi} \int_{a}^{x} B(y) d y\right) d x \underset{\kappa \rightarrow+\infty}{\longrightarrow} 0$

and so

$$
2 \exp \left(-\lambda_{F}^{\kappa}(\underline{\psi}) a\right) \underset{\kappa \rightarrow+\infty}{\longrightarrow} 1,
$$

which shows that $\liminf _{\kappa \rightarrow \infty} \lambda_{F}^{\kappa}(\psi) \geqslant \lim _{\kappa \rightarrow \infty} \lambda_{F}^{\kappa}(\underline{\psi})=\log 2 / a$, concluding the proof of the theorem.

We give a numerical illustration of this theorem in Figure 5. These numerical simulations were produced along the lines of [3] using a monotone finite difference scheme. The Floquet eigenvalue was computed by applying the power algorithm to the one day discretized evolution operator.

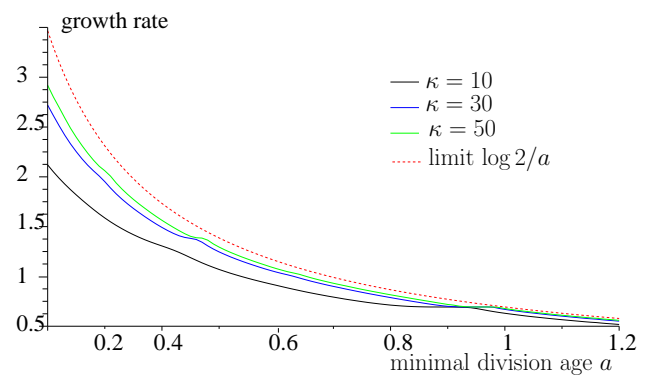

Figure 5. Convergence of the Floquet eigenvalue to $\log 2 / a$.

3.2. When $\psi$ can vanish: proof of Theorem 1.2. When $\psi$ can vanish, the conclusion of Theorem 2 fails; its proof uses the assumption that $\min (\psi)>0$. Then, we next show that more complex behaviors appear. In particular, the limit of $\lambda_{F}^{k}$ can be a discontinuous, staircase like, function of the majority age $a$, and we shall see that the limits $\kappa \rightarrow+\infty$ and $\psi \rightarrow 0$ (locally) may not commute.

3.2.1. Intuitive derivation of the formula via a deterministic jump Markov process. We consider the original PDE (2), taking $T=1$,

$$
\left\{\begin{array}{l}
\frac{\partial}{\partial t} n(t, x)+\frac{\partial}{\partial x} n(t, x)+\kappa \psi(t) \mathbb{1}_{[a, \infty[}(x) n(t, x)=0, \\
n(t, 0)=2 \kappa \psi(t) \int_{a}^{\infty} n(t, x) d x .
\end{array}\right.
$$

where $\psi$ is a 1-periodic square wave, such that if $\lfloor t\rfloor$ is the integer part of $t$,

$$
\exists 0<\tau<1, \quad \psi=t \mapsto \mathbb{1}_{[0, \tau[}(t-\lfloor t\rfloor) .
$$

We next derive the limit of the growth rate $\lambda$ when $\kappa \rightarrow+\infty$ (within this section, $\lambda$ denotes the Floquet eigenvalue $\lambda_{F}^{\kappa}$, thought of as a function of $a$ and $\psi$ ). This derivation will be purely formal for the moment, leading to an ansatz the validity of which will be proved in the next sections.

Intuitively, whenever the cell is old enough in the cycle $(x>a)$ and the time is favorable $(0 \leqslant t-\lfloor t\rfloor \leqslant \tau)$, a division may occur. The idea is to consider that at 
time $t=0$, we have only cells of age $0\left(n(0, \cdot)=\delta_{0}\right)$, and to look formally for an asymptotic solution

$$
n(t, \cdot) \sim 2^{m(t)} \delta_{\alpha(t)}, \quad \kappa \rightarrow \infty
$$

of the PDE (26), meaning that the population at time $t$ consists mostly of $2^{m(t)}$ individuals all of age $\alpha(t)$, as $\kappa \rightarrow \infty$. If for some integer time $K$, we have $\alpha(K)=0$, so that $n(K, \cdot) \sim 2^{m(K)} \delta_{0}$, using the time-periodicity and linearity of (26) we may expect that $n(p K, \cdot) \sim 2^{p m(K)} \delta_{0}$, for all integers $p \geqslant 1$. Commuting the limits as $\kappa \rightarrow \infty$ and $p \rightarrow \infty$, we arrive at the following expression for the limit of the growth rate as $\kappa \rightarrow \infty$,

$$
\lambda^{\infty}(a):=\frac{m(K)}{K \log 2}=\lim _{t \rightarrow \infty} \frac{m(t)}{t \log 2} .
$$

The idea is now that the trajectory $(\alpha(t))_{t \geqslant 0}$, representing the age of the population, is produced by a degenerate (deterministic) time-dependent càdlág jump process, in which the state space is $\mathbb{R}_{+}$, and a particle moves to the right with unit speed, untill it reaches an age and a time at which division is permitted, meaning that $\alpha(t) \geqslant a$ and $t \in[0, \tau[+\mathbb{N}$. At every time satisfying this condition, it jumps to point 0 (age is reset to zero and a division occurs). The number $m(t)$ counts the number of jumps up to time $t$ and represents the number of divisions of the cell which occured up to that time.

It suffices for our argument to consider the case in which $\alpha(0)=0$. Then, the division times can be readily computed: if we start from age 0 at time 0 , then, a division should occur at every instant $a, 2 a, \ldots, k a$ as long as it is permitted, meaning that $a, 2 a, \ldots, k a \in[0, \tau[+\mathbb{N}$. Let

$$
K_{a}:=\inf \{k \in \mathbb{N} ; k a \in[\tau, 1[+\mathbb{N}\},
$$

so that $K_{a} a$ is the first time at which a division is not permitted, or $K_{a}=\infty$. When, for the first time, we reach a possible division time $K_{a} a$ that is not permitted, then the corresponding division occurs later, namely at the next period : $\left\lceil K_{a} a\right\rceil$, where $\lceil\cdot\rceil$ denotes the upper integer part. Then, at time $\left\lceil K_{a} a\right\rceil$ the population divides (for the $K_{a}$ th time) and we have $n\left(\left\lceil K_{a} a\right\rceil, \cdot\right)=2^{K_{a}} \delta_{0}$. Therefore, (28) yields

$$
e^{\lambda^{\infty}(a)\left\lceil K_{a} a\right\rceil}=2^{K_{a}}, \quad \lambda^{\infty}(a)=\frac{K_{a}}{\left\lceil K_{a} a\right\rceil} \log 2 .
$$

When $K_{a}=\infty$, we shall adopt the convention that $\lambda^{\infty}(a)=(\log 2) / a$.

This is illustrated in Figure 3.2.1.

Working with a semi-open interval $[\tau, 1[$ in $(29)$ is consistent with the càdlág nature of the process $\alpha(t)$, however, it leads to the possibility that $K_{a}=\infty$, which is somehow unpleasant. Hence, we will use in the sequel an equivalent formulation of $\lambda^{\infty}$, obtained by replacing $[\tau, 1[$ by the closed interval $[\tau, 1]$,

$$
N_{a}:=\min \left\{k \in \mathbb{N} ; k a \in W_{\tau}\right\}, \quad W_{\tau}:=[\tau, 1]+\mathbb{N} .
$$

Lemma 3.1. We have $N_{a}<\infty$. Moreover,

$$
\lambda^{\infty}(a)=\frac{N_{a}}{\left\lceil N_{a} a\right\rceil} \log 2 .
$$

Proof. If $a$ is irrational, the finiteness of $N_{a}$ follows from the density of the sequence $k a$ modulo 1 , in $[0,1$ [; whereas if $a$ is rational, we have $k a \in 1+\mathbb{N}$ for some integer $k$, so that $N_{a} \leqslant k$ is finite. It remains to show that the expressions (30) and (31) coincide. Assume first that $N_{a} a \in\left[\tau, 1\left[+\mathbb{N}\right.\right.$. Then, by definition of $\left.N_{a}, k a \in\right] 0, \tau[+\mathbb{N}$ 


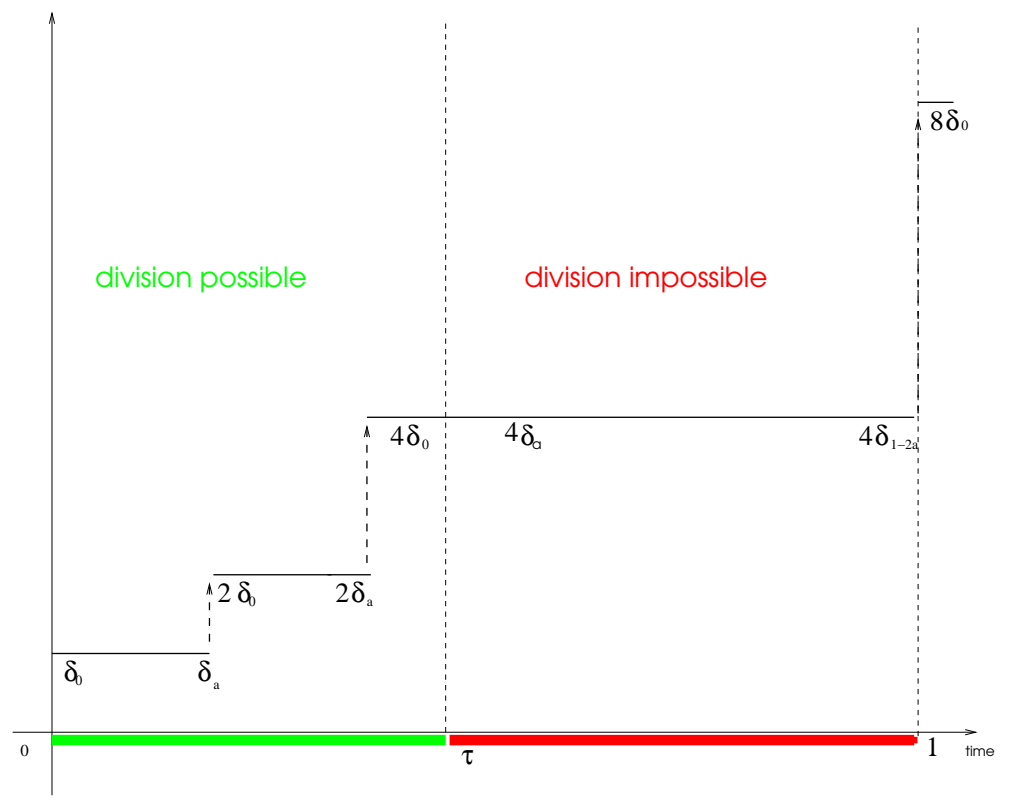

Figure 6. Example of the derivation of the formula. Here $2 a<$ $\tau<3 a<1$ and we obtain $n(1, \cdot)=8 n(0, \cdot)$. Hence we expect $\lambda^{\infty}(a)=3 \log (2)$

for $1 \leqslant k<N_{a}$, and so, $k a \in\left[0, \tau\left[\right.\right.$ for $0 \leqslant k<N_{a}$, which implies that $K_{a}=N_{a}$. Assume now that $N_{a} a \in 1+\mathbb{N}$. Then, we deduce from $\left.k a \in\right] 0, \tau\left[+\mathbb{N}\right.$, for $1 \leqslant k<N_{a}$ that $\left.\left(N_{a}+k\right) a \in\right] 0, \tau\left[+\mathbb{N}\right.$, for all $1 \leqslant k<N_{a}$, and so $K_{a}=\infty$, which by our convention regarding (30) leads to (31).

In the remaining subsections, we show that $\lim _{\kappa \rightarrow \infty} \lambda^{\kappa}(a)$ does coincide with the function $\lambda^{\infty}(a)$ (Theorem 1.2). Throughout the proof, the parameter $\tau$ will be fixed. The proof of the theorem relies on the following strategy:

(i) prove that the ansatz $\lambda^{\infty}(a)$ (staircase formula) is an upper bound for $\lambda^{\kappa}(a)$, for every $\kappa>0$, (Section 3.3);

(ii) derive technical properties of the limit and of $\lambda^{\infty}(a)$; in particular monotonicity and right continuity, (Section 3.2.2);

(iii) assume that $a$ is not the right end of a step of $\lambda^{\infty}$,

(iv) consider the generational formulation of the division equation with initial data $N^{\kappa}(0, x)$ and show that at time $\left\lceil N_{a} a\right\rceil$, almost every individual has reached generation $N_{a}$ and deduce the convergence for such an $a$.

(v) conclude that the limit of $\lambda^{\kappa}$ as $\kappa \rightarrow \infty$ is given by the staircase formula of $\lambda^{\infty}(a)$, for all $a>0$, using the fact that both $\lambda^{\infty}$ and $\lim _{\kappa \rightarrow \infty} \lambda^{\kappa}$ are right continuous functions of the parameter $a$.

3.2.2. Preliminaries: technical properties of $\lambda^{\infty}$ and of $\lim _{\kappa \rightarrow \infty} \lambda^{\kappa}$. We shall need a number of technical observations regarding the function $\lambda^{\infty}$ defined in (30).

Lemma 3.2. The function $a \mapsto \lambda^{\infty}(a)$ is nonincreasing. 
Proof. Let $(\alpha(t))_{t \geqslant 0}$ denote the trajectory of the jump process defined above, starting from $\alpha(0)=0$, and let $m(t)$ denote the number of jumps (divisions) up to time $t$ (included). We have

$$
m(t)=\sup \left\{k \in \mathbb{N} ; \exists 0 \leqslant a_{1} \leqslant \ldots \leqslant a_{k} \leqslant t, a_{i+1}-a_{i} \geqslant a, a_{i} \in[0, \tau[+\mathbb{N}\}\right.
$$

(number of divisions since time 0 )

Since the constraint $a_{i+1}-a_{i} \geqslant a$ gets stronger as $a$ increases, it is then obvious that $m$ is a nonincreasing function of $a$. We have, by definition of $\lambda^{\infty}$,

$$
\lim _{t \rightarrow+\infty} \frac{m(t)}{t}=\frac{N_{a}}{\left\lceil N_{a} a\right\rceil}=\frac{\lambda^{\infty}}{\log 2}
$$

Since for every $t, m(t)$ is a nonincreasing function of $a$, the same is true for $\lim _{t} m(t) / t=\lambda^{\infty} / \log 2$.

Lemma 3.3. The limit $\ell(a)=\lim _{\kappa \rightarrow \infty} \lambda^{\kappa}(a)$ satisfies

$$
\ell(a)=\sup _{\kappa>0} \lambda^{\kappa}(a)
$$

and the map $a \mapsto \ell(a)$, is nonincreasing and right continuous.

Proof. Since $\lambda^{\kappa}(a) \leqslant \log 2 / a$, and since, by the comparison principle (Theorem 1.1), $\lambda^{\kappa}(a)$ is a nondecreasing function of $\kappa$,

$$
\ell(a)=\sup _{\kappa>0} \lambda^{\kappa}(a) \leqslant \frac{\log 2}{a} .
$$

Furthermore, since for any fixed $\kappa, \lambda^{\kappa}(a)$ is nonincreasing with $a$, so is $\ell(a)$. Finally, since for any fixed $\kappa>0, \lambda^{\kappa}(a)$ is continuous with respect to $a, \ell(a)$, which is a supremum of a family of lower semi-continuous functions, is lower semi-continuous. Combined with monotonicity, this leads to the convenient property:

$$
\forall a>0, \quad \ell(a)=\liminf _{x \rightarrow a} \ell(x)=\lim _{x \rightarrow a+0} \ell(a) .
$$

That is, $\ell(a)=\lim _{\kappa \rightarrow \infty} \lambda^{\kappa}(a)$ is right continuous.

Lemma 3.4. The function $a \mapsto \lambda^{\infty}(a)$ is right continuous.

Proof. By Lemma 3.1,

$$
N_{a} a=\min W_{\tau} \cap \mathbb{N} a=: f(a), \quad \lambda^{\infty}(a)=\frac{N_{a}}{\left\lceil N_{a} a\right\rceil} \log 2=\frac{f(a)}{a\lceil f(a)\rceil} \log 2,
$$

where we just denote $f(a)=N_{a} a$. To show that the function $\lambda^{\infty}$ is right continuous, it suffices to prove that

$$
g(a)=\frac{f(a)}{\lceil f(a)\rceil},
$$

is right continuous. If $g(a) \neq 1$, this means that $f(a) \neq\lceil f(a)\rceil$, and therefore, for $\varepsilon>0$ small enough, we can have $n(a+\varepsilon) \in\left[\lfloor n a\rfloor,\lfloor n a\rfloor+\tau\right.$ [ for all $n<N_{a}$ and $N_{a}=N_{a+\varepsilon}, f(a+\varepsilon)=f(a)+N_{a} \varepsilon$ and $\lceil f(a)\rceil=\lceil f(a+\varepsilon)\rceil$. Thereby, for $\varepsilon>0$ small enough, we have $g(a+\varepsilon)=g(a)+\frac{N_{a}}{\lceil f(a)\rceil} \varepsilon$, which leads to the right continuity of $g$ at $a$. If $g(a)=1$, then, we can check that

$$
\lim _{\varepsilon \rightarrow 0^{+}} f(a+\varepsilon)=+\infty
$$

and so

$$
\lim _{\varepsilon \rightarrow 0^{+}} g(a+\varepsilon)=1
$$


These observations leads to the following useful property (stating basically that $\lambda^{\infty}$ is piecewise constant)

Lemma 3.5. For all $a>0, I_{a}=\left\{\lambda^{\infty}(x)=\lambda^{\infty}(a)\right\}$ is an interval with nonempty interior. Moreover, inf $I_{a} \in I_{a}$. Finally, if we denote

$$
E_{\tau}:\left\{a>0, a=\sup I_{a}\right\},
$$

we have

- if $a \in E_{\tau}$ then $\lambda^{\infty}$ is continuous at $a$,

- if $a \in E_{\tau}$ then $N_{a}$ a is an integer.

We shall see that the set $E_{\tau}$ contains exactly the right ends of steps of $\lambda^{\infty}$ where no jump occur. An infinite number of steps accumulate at the right of every point of $E_{\tau}$. On Figure 1, we can see for instance that $1 / 2$ belongs to $E_{2 / 3}$

Proof. Step 1: $I_{a}$ is an interval

Firstly, this set is necessary an interval by monotonicity : if $a^{\prime} \in I_{a}$, then for any convex combination of $a, a^{\prime}$, we have

$$
\lambda^{\infty}(a)=\lambda^{\infty}\left(\max \left(a, a^{\prime}\right)\right) \leqslant \lambda^{\infty}\left(\theta a+(1-\theta) a^{\prime}\right) \leqslant \lambda^{\infty}\left(\max \left(a, a^{\prime}\right)\right)=\lambda^{\infty}(a) .
$$

To prove that it is nontrivial, we use the definition of $N_{a}$. By definition $a, 2 a, \ldots\left(N_{a}-\right.$ 1) $a \notin W_{\tau}$. As $W_{\tau}$ is a closed set, its complementary is open and thus for $\varepsilon>0$ small enough,

]$a-\varepsilon, a+\varepsilon\left[\cap W_{\tau}=\right] 2(a-\varepsilon), 2(a+\varepsilon)\left[\cap W_{\tau}=\right]\left(N_{a}-1\right)(a-\varepsilon),\left(N_{a}-1\right)(a-\varepsilon)\left[\cap W_{\tau}=\emptyset\right.$.

Finally, for $\varepsilon>0$ small enough, we have necessarily $\left.] N_{a}(a-\varepsilon), N_{a} a\right] \subset W_{\tau}$ or $\left[N_{a} a, N_{a}(a+\varepsilon)\left[\subset W_{\tau}\right.\right.$ (possibly both). Thereby, for any $a$, there exists $\varepsilon>0$ such that $[a, a+\varepsilon[$ or $] a-\varepsilon, a]$ is contained in $I_{a}$ which has then a nonempty interior (contains an interval of size $\varepsilon$ ). The fact that inf $I_{a}$ belongs to $I_{a}$ is just a consequence of right continuity.

Step 2: continuity on $E_{\tau}$

The continuity follows from right continuity of $\lambda^{\infty}$ and the fact that in this case, $\lambda^{\infty}\left(\sup I_{a}\right)=\lim _{x \rightarrow \sup I_{a}-0} \lambda^{\infty}(x)$, that is $\lambda^{\infty}$ is also left continuous.

Step 3: $N_{a} a=\left\lceil N_{a} a\right\rceil$, forall $a \in E_{\tau}$

Assume that $a$ satisfies $\left\lceil N_{a} a\right\rceil-1+\tau \leqslant N_{a} a<\left\lceil N_{a} a\right\rceil$. Then, for some $\varepsilon>0$, we have $\left\lceil N_{a} a\right\rceil-1+\tau \leqslant N_{a} a^{\prime}<\left\lceil N_{a} a\right\rceil$ for any $\left.a^{\prime} \in\right] a, a+\varepsilon[$. Thereby, we can claim by definition of $N_{a}$ that

$$
N_{a^{\prime}} \leqslant N_{a}, \quad\left\lceil N_{a^{\prime}} a^{\prime}\right\rceil \leqslant\left\lceil N_{a} a\right\rceil .
$$

Suppose now that in addition that $a \in E_{\tau}$. By definition, as $a=\sup I_{a}$, we have $\lambda^{\infty}\left(a^{\prime}\right) \neq \lambda^{\infty}(a)$ for $\left.a^{\prime} \in\right] a, a+\varepsilon\left[\right.$. Furthermore for $\left.a^{\prime} \in\right] a, a+\varepsilon\left[, \lambda^{\infty}\right.$ takes its values in

therefore, for $\left.a^{\prime} \in\right] a, a+\varepsilon[$, we have

$$
\left\{\frac{N}{p} \log 2, \quad N \leqslant N_{a}, \quad p \leqslant\left\lceil N_{a} a\right\rceil\right\},
$$

$$
\left|\lambda^{\infty}(a)-\lambda^{\infty}\left(a^{\prime}\right)\right| \geqslant \frac{1}{\left(\left\lceil N_{a} a\right\rceil\right)^{2}} \log 2
$$

and this contradicts the continuity of $\lambda^{\infty}$ at $a$. 
The set $E_{\tau}$ has a last interesting property.

Lemma 3.6. The set $E_{\tau}$ is discrete.

Proof. Let $a \in E_{\tau}$. Thanks to Lemma 3.5, we know that $\lambda^{\infty}$ is constant on $I_{a}$ and therefore $a^{\prime} \notin E_{\tau}$ for $\left.a^{\prime} \in\right] \inf I_{a}, a[$. It remains to prove the existence of $\varepsilon>0$ such that $] a, a+\varepsilon\left[\cap E_{\tau}=\emptyset\right.$.

To prove this, let $m \in \mathbb{N} \backslash\{0\}$ satisfy $\tau \leqslant 1-\frac{1}{m}$. Choose $\varepsilon=\frac{1}{2 N_{a} m}$. Let $\left.a^{\prime}=a+\varepsilon^{\prime} \in\right] a, a+\varepsilon\left[\right.$. We have $0<\varepsilon^{\prime}<\varepsilon$. For every integer $k$, we have

$$
k N_{a} a^{\prime}=k N_{a} a+k N_{a} \varepsilon^{\prime} .
$$

As $\varepsilon^{\prime} \leqslant \frac{1}{2 N_{a}}$, there exists an integer $k$ such that $k N_{a} \varepsilon^{\prime} \leqslant 1<(k+1) N_{a} \varepsilon^{\prime}$. We have then,

$$
\tau \leqslant 1-\frac{1}{m} \leqslant 1-2 N_{a} \varepsilon^{\prime}<(k-1) N_{a} \varepsilon^{\prime}<k N_{a} \varepsilon^{\prime} \leqslant 1 .
$$

This leads to, adding $(k-1) N_{a} a$,

$$
(k-1) N_{a} a+\tau<(k-1) N_{a} a^{\prime}<(k-1) N_{a} a+1 .
$$

Since $N_{a} a \in \mathbb{N}$, this means that $(k-1) N_{a} a^{\prime} \in \operatorname{int}\left(W_{\tau}\right)$. If we assume that $a^{\prime} \in E_{\tau}$, we have $N_{a^{\prime}} a^{\prime} \in \mathbb{N}$ and then by construction

$$
\left\{0, a^{\prime}, \ldots, N_{a^{\prime}} a^{\prime}\right\} \cap W_{\tau}=\left\{0, N_{a^{\prime}} a^{\prime}\right\}
$$

It follows then that

$$
a^{\prime} \mathbb{N} \cap W_{\tau}=\left(N_{a^{\prime}} a^{\prime}\right) \mathbb{N},
$$

which in particular implies $a^{\prime} \mathbb{N} \cap \operatorname{int}\left(W_{\tau}\right)=\emptyset$. This contradicts the existence of $p$.

We have proved that

$$
] \inf I_{a}, a+\varepsilon\left[\cap E_{\tau}=\{a\}\right.
$$

which ends the proof of Lemma 3.6

3.3. Upper bound on $\lambda^{\kappa}$. We next show that $\lambda^{\infty}$ is an upper bound of $\lambda^{\kappa}$, for each $\kappa>0$. This will turn out to be a relatively simple consequence of the heuristic argument, involving the earliest possible times for division, which led to the formula of $\lambda^{\infty}$. The proof relies the generational reformulation of the division equation. Let $a>0, \tau \in] 0,1[, \kappa>0$ be fixed. We take the eigenvector as an initial data.

$$
\left\{\begin{array}{l}
\partial_{t} n_{i}+\partial_{x} n_{i}+\kappa \psi(t) \mathbb{1}_{[a, \infty[}(x) n_{i}(t, x)=0, \\
n_{i+1}(t, 0)=\kappa \psi(t) \int_{a}^{\infty} n_{i}(t, x) d x, \\
n_{0}(x)=N^{\kappa}(0, x) .
\end{array} .\right.
$$

We note by $I_{\max }(t)$ the maximal integer $i$ (generation) such that $n_{i}(t, \cdot) \not \equiv 0$. At time $t=0$, we have $I_{\max }(0)=0$. Since cells need to have an age bigger than $a$ to change generation, we have necessarily $I_{\max }(t) \leqslant 1$ for $t<a$ and more generally,

$$
\begin{gathered}
I_{\max }(t) \leqslant n, \quad \text { if } t<n a . \\
I_{\max }(t) \leqslant N_{a} \quad \text { if } t<\left(N_{a}\right) a .
\end{gathered}
$$

We introduce the notation $p_{a}=\left\lceil N_{a} a\right\rceil-1$. The integers $N_{a}, p_{a}$ are then the minimal integers such that

$$
p_{a}+\tau \leqslant N_{a} a \leqslant p_{a}+1 .
$$


We use now the properties of the equation: since no change of generation may occur between times $p_{a}+\tau$ and $p_{a}+1$,we have

$$
I_{\max }(t) \leqslant N_{a} \quad \text { if } p_{a}+\tau \leqslant t<p_{a}+1 .
$$

Now, from the definition of $N^{\kappa}$ and $\lambda:=\lambda^{\kappa}$, we have

$$
\sum_{i=0}^{\infty} 2^{i} n_{i}(t, x)=e^{\lambda t} N^{\kappa}(t, x)
$$

In particular, for $t<p_{a}+1$, we have

$$
\sum_{i=0}^{I_{\max }(t)} 2^{i} n_{i}(t, x)=\sum_{0}^{N_{a}} 2^{i} n_{i}(t, x)=e^{\lambda t} N^{\kappa}(t, x) .
$$

We integrate with respect to $x$ and obtain

$$
e^{\lambda t} \int_{0}^{\infty} N^{\kappa}(t, x) d x=\sum_{0}^{N_{a}} 2^{i} \int_{0}^{\infty} n_{i}(t, x) d x \leqslant 2^{N_{a}} \sum_{0}^{N_{a}} \int_{0}^{\infty} n_{i}(t, x) d x .
$$

But we also have from the properties of the generational formulation and the definition of $I_{\max }$,

$$
\sum_{0}^{N_{a}} \int_{0}^{\infty} n_{i}(t, x) d x=\sum_{0}^{\infty} \int_{0}^{\infty} n_{i}(t, x) d x=\int_{0}^{\infty} n_{0}(0, x) d x=\int_{0}^{\infty} N^{\kappa}(0, x) d x .
$$

Combining (33-34), we get

$$
e^{\lambda t} \int_{0}^{\kappa} N^{\kappa}(t, x) d x \leqslant 2^{N_{a}} \int_{0}^{\infty} N^{\kappa}(0, x) d x, \quad t<p_{a}+1 .
$$

As $\kappa$ is fixed, we can use the continuity of $\int_{0}^{\infty} N^{\kappa}(t, x) d x$. We have therefore

$$
\exp \left(\lambda^{\kappa}\left(p_{a}+1\right)\right) \int_{0}^{\infty} N^{\kappa}\left(p_{a}+1, x\right) d x \leqslant 2^{N_{a}} \int_{0}^{\infty} N^{\kappa}(0, x) d x
$$

As $N^{\kappa}\left(p_{a}+1, x\right)=N^{\kappa}(0, x)$, we have

$$
\exp \left(\lambda^{\kappa}\left(p_{a}+1\right)\right) \leqslant 2^{N_{a}} .
$$

And therefore, as expected

$$
\lambda^{\kappa}(a) \leqslant \frac{N_{a}}{p_{a}+1} \log 2=\lambda^{\infty}(a) .
$$

\subsection{Bounding $\lambda$ from below.}




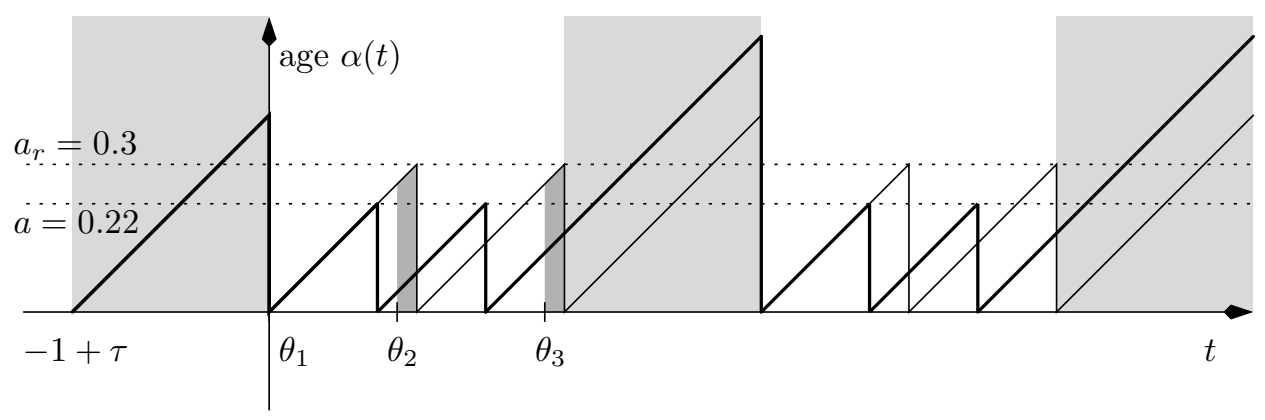

Figure 7. Illustration of Lemma 3.7 and of Lemma 3.15: the trajectory of the jump process $\alpha(t)$ starting from $\alpha(-1+\tau)=0$ is shown in bold for $a=0.22$ and $\tau=0.6$ (division is not permitted in the light ray regions). Here, $N_{a}=3$ and $p_{a}=0$, so $\lambda^{\infty}(a)=$ $3 \log 2$. The minimal division age $a$ can be increased up to $a_{r}=$ 0.3 , leaving $\lambda^{\infty}(a)$ unchanged. The conditions of Lemma 3.7 can be satisfied with a positive $\varepsilon$, because, for all $a^{\prime} \in\left[a, a_{r}[\right.$, the combinatorial type of the trajectory of the jump process $\alpha(t)$ is unchanged (trajectory shown by a thin line, for $a^{\prime}$ close to $a_{r}$ ); choosing $\theta_{1}:=0$, the times $\theta_{2}$ and $\theta_{3}$ can be taken to be the abscissæ of the left of the dark grey regions of width $\varepsilon<\left(a_{r}-\right.$ a) $/ 2=0.04$.

Step 1: Deriving inequality (12). (with $\varepsilon$ instead of $r$.) We derive the bound through the following technical lemma, in which the sequence $\theta_{1}, \ldots, \theta_{k}$ represents possible successive division times of an individual of age $1-\tau$ at time 0 .

Lemma 3.7. Suppose there exist $\theta_{1}, \ldots \theta_{N_{a}}$ and $\varepsilon \geqslant 0$, satisfying the following properties:

$$
\left\{\begin{array}{l}
\theta_{1} \geqslant \max (a-1+\tau, 0), \\
\forall i \leqslant N_{a}-1, \quad \theta_{i+1}-\left(\theta_{i}+\varepsilon\right) \geqslant a, \\
\forall i \in\left\{1, \ldots, N_{a}\right\} \quad \theta_{i}+\varepsilon<\left\lfloor\theta_{i}\right\rfloor+\tau,
\end{array}\right.
$$

then the following inequality holds:

$$
e^{\lambda^{\kappa}(a)\left(p_{a}+1\right)} \geqslant\left(2-e^{-\kappa \varepsilon}\right)^{N_{a}} .
$$

Intuitively, this lemma shows that if there is a "tube" of positive width $\varepsilon$, limited from below by such a sequence $\theta_{1}, \ldots, \theta_{N_{a}}$ and included in the set of times at which division is permitted, then, "enough" individuals can follow this tube, so the Floquet eigenvalue $\lambda^{\kappa}(a)$ can be bounded below by $\left(N_{a} \log 2\right) /\left(p_{a}+1\right)$, up to a correction of order $O(\exp (-\kappa \epsilon))$ as $\kappa \rightarrow \infty$.

The existence of the sequence $\theta_{1}, \ldots, \theta_{N_{a}}$ will be established in Step 2 below when $\varepsilon=0$. We shall see in Step 3 that such a sequence does exist, for some $\varepsilon>0$, provided that $a$ does not coincide with the right end $a_{r}$ of a step of the staircase function $\lambda^{\kappa}$. This is illustrated in Figure 7 .

Proof. As for the upper bound, we consider the generational reformulation 16, in which $K(t, x)=\kappa \psi(t) \mathbb{1}_{[a, \infty[}[x)$ and the eigenvector $N^{\kappa}(t=0, x)$ is the initial data. 
From the definition, we have

$$
\sum_{i} 2^{i} n_{i}\left(p_{a}+1, x\right)=N^{\kappa}(0, x) \exp \left(\lambda^{\kappa}(a)\left(p_{a}+1\right)\right) .
$$

Especially,

$$
\sum_{i} 2^{i} \int_{0}^{\infty} n_{i}\left(p_{a}+1, x\right) d x=\exp \left(\lambda^{\kappa}(a)\left(p_{a}+1\right)\right) \int_{0}^{\infty} N^{\kappa}(0, x) d x .
$$

We recall that $N^{\kappa}(0, x)=0$ for $x \in[0,1-\tau[$. As we look for a bound from below, thanks to the comparison principle, it is sufficient to consider the individuals that are the youngest at the beginning, that is the individuals starting at age $(1-\tau)$. As $K(t, x)$ is nondecreasing with respect to $x$, we know that if we choose $K^{\prime}(t, x) \leqslant K(t, x)$, and $n_{i}^{\prime}$ with obvious notations, then we have thanks to the comparison principle

$$
\sum_{i} 2^{i} \int_{0}^{\infty} n_{i}^{\prime}\left(p_{a}+1, x\right) d x \leqslant \exp \left(\lambda^{\kappa}(a)\left(p_{a}+1\right)\right) \int_{0}^{\infty} N^{\kappa}(0, x) d x .
$$

We choose

$$
K^{\prime}(t, x)= \begin{cases}\kappa & \text { if } \left.t \in] \theta_{1}, \theta_{1}+\varepsilon\right], \quad x \geqslant a \\ \kappa & \text { if } \left.t \in] \theta_{i}+(i-1) \varepsilon, \theta_{i}+i \varepsilon\right], \quad x \geqslant a, \\ 0 & \text { otherwise. }\end{cases}
$$

From the definition of $\left(\theta_{1}, \ldots \theta_{N_{a}}\right)$ and $\varepsilon$, we have $K^{\prime}(t, x) \leqslant K(t, x)$. The most important property of the transition rate $K^{\prime}$ is the following.

Lemma 3.8. Suppose that $n_{i}^{\prime}$ are defined by the generational dynamics with transition rate $K^{\prime}$, then for any $1 \leqslant i \leqslant N_{a}$,

$$
\operatorname{supp} n_{i}^{\prime}\left(\theta_{i}, \cdot\right) \subset[a,+\infty[.
$$

Proof. We have for $\left.\left.x<a, \theta_{i}-x \notin \bigcup_{i}\right] \theta_{i}+(i-1) \varepsilon, \theta_{i}+i \varepsilon\right]$ and thereby $K^{\prime}\left(\theta_{i}-x, \cdot\right)=$ 0 . Therefore for any $j \geqslant 0$

$$
n_{j+1}^{\prime}\left(\theta_{i}-x, 0\right)=\int_{0}^{\infty} K^{\prime}\left(\theta_{i}-x, y\right) n_{j}^{\prime}\left(\theta_{i}-x, y\right) d y=0,
$$

and using the characteristics

$$
n_{j+1}\left(\theta_{i}, x\right)=n_{j+1}\left(\theta_{i}-x, 0\right) e^{-\int_{0}^{x} K^{\prime}\left(\theta_{i}-x+s, s\right) d s}=0 .
$$

Finally, as $\operatorname{supp} n_{0}^{0} \subset\left[1-\tau,+\infty\left[\right.\right.$ and $\operatorname{as} \operatorname{supp} n_{0}(t, \cdot)=\operatorname{supp} n_{0}^{0}+t$, we have since $\theta_{i} \geqslant a-1+\tau$ for any $i$

$$
\operatorname{supp} n_{0}\left(\theta_{i}, \cdot\right) \subset[a,+\infty[\text {. }
$$

The latter inclusion means that for any $j>0$, at time $\theta_{j}$, every individual is mature enough to divide.

Lemma 3.9. The population $n_{i}$ satisfies the following equality : defining $I_{i}(t)=$ $\int_{0}^{\infty} n_{i}(t, x) d x$, we have

$$
\forall j>0, \forall i, \quad\left\{\begin{array}{l}
I_{0}\left(\theta_{j}+\varepsilon\right)=e^{-\kappa \varepsilon} I_{0}\left(\theta_{j}\right), \\
I_{i+1}\left(\theta_{j}+\varepsilon\right)=e^{-\kappa \varepsilon} I_{i+1}\left(\theta_{j}\right)+\left(1-e^{-\kappa \varepsilon}\right) I_{i}\left(\theta_{j}\right) .
\end{array}\right.
$$


Proof. It is essentially based on the previous lemma. By construction, we have $\varepsilon<a$. Since we already proved that $\operatorname{supp} n_{i}\left(\theta_{j}, \cdot\right) \subset[a,+\infty[$, we can claim

$$
\operatorname{supp} n_{i}\left(\theta_{j}+\varepsilon, \cdot\right) \subset[0, \varepsilon] \cup\left[a+\varepsilon,+\infty\left[\text { for } i \geqslant 1, \operatorname{supp} n_{0}\left(\theta_{j}+\varepsilon\right) \subset[a+\varepsilon,+\infty[\right.\right.
$$

From the definition of the $n_{j}$, we have,

$\forall i, \forall x \geqslant a+\varepsilon, \quad n_{i}\left(\theta_{j}+\varepsilon, x\right)=n_{i}\left(\theta_{j}, x-\varepsilon\right) e^{-\int_{0}^{\varepsilon} K^{\prime}\left(\theta_{j}+s, x-\varepsilon+s\right) d s}=n_{i}\left(\theta_{j}, x-\varepsilon\right) e^{-\kappa \varepsilon}$.

Therefore,

$$
\int_{x \geqslant a+\varepsilon} n_{i}\left(\theta_{j}+\varepsilon, x\right) d x=e^{-\kappa \varepsilon} \int_{x \geqslant a} n_{i}\left(\theta_{j}, x\right) d x=e^{-\kappa \varepsilon} I_{i}\left(\theta_{j}\right) .
$$

We have also, for all $i \geqslant 0$,

$$
\begin{aligned}
\int_{x \leqslant \varepsilon} n_{i+1}\left(\theta_{j}+\varepsilon, x\right) d x & =\int_{0}^{\varepsilon} n_{i+1}\left(\theta_{j}+\varepsilon-x, 0\right) e^{-\int_{0}^{x} K^{\prime}\left(\theta_{j}+\varepsilon-x+s, s\right) d s} d x \\
& =\int_{0}^{\varepsilon} n_{i+1}\left(\theta_{j}+\varepsilon-x, 0\right) d x \\
& =\int_{0}^{\varepsilon} n_{i+1}\left(\theta_{j}+s, 0\right) d x .
\end{aligned}
$$

Since $\operatorname{supp} n_{i}\left(\theta_{j}, \cdot\right) \subset[a, \infty[$ and $\varepsilon<a$, we also have

$n_{i+1}\left(\theta_{j}+s, 0\right)=\int_{0}^{\infty} K^{\prime}\left(\theta_{j}+s, x\right) n_{i}\left(\theta_{j}+s, x\right) d x=\int_{a}^{\infty} \kappa n_{i}\left(\theta_{j}, x\right) e^{-\kappa s}=I_{i}\left(\theta_{j}\right) \kappa e^{\kappa s}$.

We integrate and obtain finally

$$
\int_{x \leqslant \varepsilon} n_{i+1}\left(\theta_{j}+\varepsilon, x\right) d x=\left(1-e^{-\kappa \varepsilon}\right) I_{i}\left(\theta_{j}\right)
$$

This completes the proof of the lemma.

To conclude the proof of Lemma 3.7, we shall need the following binomial type representation.

Lemma 3.10. Denote by $\left(\begin{array}{l}n \\ p\end{array}\right)$ the binomial coefficients (with value 0 if $p>n$ ), then we have

$$
\forall i, I_{i}\left(\theta_{j}+\varepsilon\right)=I_{0}(0)\left(\begin{array}{c}
j \\
i
\end{array}\right)\left(1-e^{\kappa \varepsilon}\right)^{i}\left(e^{-\kappa \varepsilon}\right)^{j-i} .
$$

Proof. This is readily obtained by induction from Lemma 3.9, using the fact that $I_{i}\left(\theta_{j}+\epsilon\right)=I_{i}\left(\theta_{j+1}\right)$, for all $j$.

We finally estimate

$$
\sum_{i} 2^{i} \int_{0}^{\infty} n_{i}^{\prime}\left(p_{a}+1, x\right) d x=\sum_{i} 2^{i} I_{i}\left(p_{a}+1\right) .
$$

It follows from the construction of $K^{\prime}$ that $I_{i}\left(p_{a}+1\right)=I_{i}\left(\theta_{N_{a}}+\varepsilon\right)$. Therefore,

$$
\begin{aligned}
\sum_{i} 2^{i} \int_{0}^{\infty} n_{i}^{\prime}\left(p_{a}+1, x\right) d x & =I_{0}(0) \sum_{i} 2^{i}\left(\begin{array}{c}
N_{a} \\
i
\end{array}\right)\left(1-e^{\kappa \varepsilon}\right)^{i}\left(e^{-\kappa \varepsilon}\right)^{N_{a}-i} \\
& =\left(2\left(1-e^{-\kappa \varepsilon}\right)+e^{-\kappa \varepsilon}\right)^{N_{a}}=\left(2-e^{-\kappa \varepsilon}\right)^{N_{a}} .
\end{aligned}
$$

This ends the proof of Lemma 3.7. 
Step 2: Construction of the sequence $\theta_{i}$, with $\varepsilon=0$. We need now to prove the existence of the sequence $\theta_{i}$ used in Lemma 3.7. First we prove the following

Lemma 3.11. There exists a sequence $\theta_{i}$ as in Lemma 3.7 with $\varepsilon=0$.

The following $t_{k}$ 's define the dynamics of successive division times of a cell initially of age $x$ changing generation/dividing whenever it reaches age $a$ at a time which is allowed. These will be used afterwards through the construction of $\theta_{i}$.

$$
\begin{aligned}
t_{0}(x)=0, \quad t_{1}(x)=\left\{\begin{array}{l}
0 \text { if } x \geqslant a, \\
a-x \text { if } a-x \notin[\tau, 1[+\mathbb{N}, \\
\lceil a-x\rceil \text { otherwise }
\end{array}\right. \\
t_{i+1}(x)=\left\{\begin{array}{l}
t_{i}(x)+a \text { if } t_{i}(x) \notin[\tau, 1[+\mathbb{N}, \\
\left\lceil t_{i}(x)+a\right\rceil \text { otherwise }
\end{array}\right.
\end{aligned}
$$

We now describe the behaviour of the $t_{k}$ 's. First of all, from the definition of $N_{a}, p_{a}$, we have the following

Lemma 3.12. The sequence $t_{i}$ satisfies the following properties :

- for $i<N_{a}, t_{i}(0)=i a, t_{N_{a}}(0)=p_{a}+1$,

- for $x>a$, for any $i \geqslant 0, t_{i+1}(x)=t_{i}(0)$,

- $x \mapsto t_{i}(x)$ is nonincreasing for any $i$,

- $\forall i, t_{i}(x) \leqslant t_{i}(0) \leqslant t_{i+1}(x)$.

Proof. The first point is an immediate consequence of the construction of $N_{a}, p_{a}$. The second point follows from the fact that if $x \geqslant a$, then $t_{1}(x)=0$, and of the definition of $N_{a}$. The last two points are straightforward.

An important property of the sequence $t_{i}$ is given in the

Lemma 3.13. Suppose $x>1-\tau$ or $\left(x=1-\tau\right.$ and $\left.N_{a} a \neq p_{a}+1\right)$, then we have

$$
t_{N_{a}}(x)<p_{a}+1 \text {. }
$$

Proof. Firstly, this is obvious if $x \geqslant a$ since in this case $t_{N_{a}}(x)=t_{N_{a}-1}(0)$. If $x<a$, we use the following

Lemma 3.14. Suppose $x<a$, let the $t_{i}(x)$ be defined by (37). Define

$$
i_{0}:=\inf _{t_{i}(x) \in \mathbb{N}} i \text {. }
$$

Then we have

$$
\begin{aligned}
\forall n<N_{a}, & t_{i_{0}+n}(x)=t_{i_{0}}(x)+n a, \\
\forall i<i_{0}, & t_{i}(x)=i a-x, \\
t_{i_{0}}(x)=\left\lceil i_{0} a-x\right\rceil &
\end{aligned}
$$

Proof. This follows immediately from the definition.

As a consequence of this lemma, we know that if $i_{0}<N_{a}$ (taking $n=N_{a}-i_{0}$ in the lemma) or if $i_{0}>N_{a}$, we have

$$
t_{N_{a}}(x) \notin \mathbb{N} \text { and } t_{N_{a}}(x) \leqslant t_{N_{a}}(0) \in \mathbb{N},
$$

therefore, in this case $t_{N_{a}}(x)<t_{N_{a}}(0)=p_{a}+1$.

If $i_{0}=N_{a}$, then, since $x>1-\tau$,

$$
N_{a} a-x \leqslant p_{a}+1-x<p_{a}+\tau .
$$

Therefore, we have necessarily $t_{N_{a}}(x)<p_{a}+\tau<p_{a}+1$ which ends the proof of Lemma 3.13 and thereby of Lemma 3.11. 
Step 3: Construction of the sequence $\theta_{i}$, with $\varepsilon>0$. Here, we use the notation $a_{r}=\sup \left\{a^{\prime}, \lambda^{\infty}\left(a^{\prime}\right)=\lambda^{\infty}(a)\right\}$ and $a_{l}=\inf \left\{a^{\prime}, \lambda^{\infty}\left(a^{\prime}\right)=\lambda^{\infty}(a)\right\}$.

Lemma 3.15. Suppose there exists a sequence $\theta_{1}, \ldots, \theta_{N_{a}}$ and $\frac{a_{r}-a}{2} \geqslant \varepsilon \geqslant 0$, such that

$$
\left\{\begin{array}{l}
\theta_{1} \geqslant \max (a-1+\tau, 0) \\
\forall i,\left[\theta_{i}, \theta_{i}+\varepsilon\right] \subset\left[\left\lfloor\theta_{i}\right\rfloor,\left\lfloor\theta_{i}\right\rfloor+\tau\right] \\
\forall i<N_{a}, \theta_{i+1} \geqslant \theta_{i}+\varepsilon+a
\end{array}\right.
$$

Then there exists a sequence $\theta_{1}^{\prime}, \ldots, \theta_{N_{a}}^{\prime}$

$$
\left\{\begin{array}{l}
\theta_{1}^{\prime} \geqslant \max (a-1+\tau, 0), \\
\forall i,\left[\theta_{i}^{\prime}, \theta_{i}^{\prime}+\varepsilon^{\prime}\right] \subset\left[\left\lfloor\theta_{i}^{\prime}\right\rfloor,\left\lfloor\theta_{i}^{\prime}\right\rfloor+\tau\right], \\
\forall i<N_{a}, \theta_{i+1}^{\prime} \geqslant \theta_{i}^{\prime}+\varepsilon^{\prime}+a^{\prime} .
\end{array}\right.
$$

with $\varepsilon^{\prime}=\min \left(\varepsilon+\frac{a-a^{\prime}}{2}, \tau\right)$.

Proof. We start the proof with an obvious but necessary remark : if $\lambda^{\infty}$ is constant on $] a_{l}, a_{r}[$, then

$$
\begin{aligned}
& \text { - } \varepsilon \leqslant \tau \\
& \text { - } a_{r}-a_{l} \leqslant \tau
\end{aligned}
$$

We build the sequence $\theta_{i}^{\prime}$ following this procedure :

$$
\theta_{i}^{\prime}=\max \left(\left\lfloor\theta_{i}\right\rfloor, \theta_{i}-\frac{a-a^{\prime}}{2}\right) .
$$

With this construction, we have $\theta_{1}^{\prime} \geqslant a^{\prime}-1+\tau$ and since $\varepsilon^{\prime}-\frac{a-a^{\prime}}{2} \leqslant \varepsilon$,

$$
\theta_{i}^{\prime}+\varepsilon^{\prime} \leqslant \max \left(\left\lfloor\theta_{i}\right\rfloor+\varepsilon^{\prime}, \theta_{i}+\varepsilon\right) \leqslant\left\lfloor\theta_{i}\right\rfloor+\tau .
$$

Note that we also have $\theta_{i}^{\prime}+\varepsilon^{\prime} \leqslant \theta_{i}+\varepsilon+\frac{a-a^{\prime}}{2}$. This helps us to check the last property:

$$
\theta_{i+1}^{\prime}-\theta_{i}^{\prime}-a^{\prime}-\varepsilon^{\prime} \geqslant \theta_{i+1}-\frac{a-a^{\prime}}{2}-\theta_{i}-a^{\prime}-\varepsilon-\frac{a-a^{\prime}}{2}=\theta_{i+1}-\theta_{i}-\varepsilon \geqslant 0 .
$$

This ends the proof of the lemma.

To complete the proof of the theorem, we combine the previous arguments. If $\lambda^{\infty}$ is constant on $\left[a_{l}, a_{r}\left[\right.\right.$, then for $a<a_{r}$ we can build a sequence $\theta_{i}$ as in Lemma 3.7 for any $\varepsilon<\left(a_{r}-a / 2\right)$ (we first build a sequence for $a_{r}-0$ and $\varepsilon=0$ and then use Lemma 3.15 to build a sequence with $\left.\varepsilon=a_{r}-a-0\right)$. Using Lemma 3.7, we get the inequality (36) for any $\varepsilon<\left(a_{r}-a / 2\right)$. By a standard continuity argument, the same is true for $\varepsilon=\left(a_{r}-a / 2\right)=r(a, \tau)$ as defined in (12). Finally, taking the logarithm of (36) with $\varepsilon=r(a, \tau)$, we get

$$
\lambda^{\kappa}(a) \geqslant \lambda^{\infty}(a) \frac{\log \left(2-e^{-\kappa r(a, \tau}\right)}{\log 2} .
$$

This leads to (12) thanks to the following inequality, which is a consequence of the concavity of the function log:

$$
\forall x \in[0,1], \quad \log (2-x) \geqslant(\log 2)(1-x) .
$$

This ends the proof of the theorem. 
3.5. Numerical illustrations. We next illustrate Theorem 1.2 by numerical experiments, that we produced using again monotone finite difference schemes (see the discussion concerning Figure 8 above). It should be noted that the bound of the rate of convergence given in this theorem vanishes when $N_{a} a$ is a multiple of the period $T$. This is confirmed by the numerical experiments: the corresponding values of $a$ are discontinuity points of the staircase function $\lambda^{\infty}(a)$, and the convergence is seen to be slow at the left of each of these points.

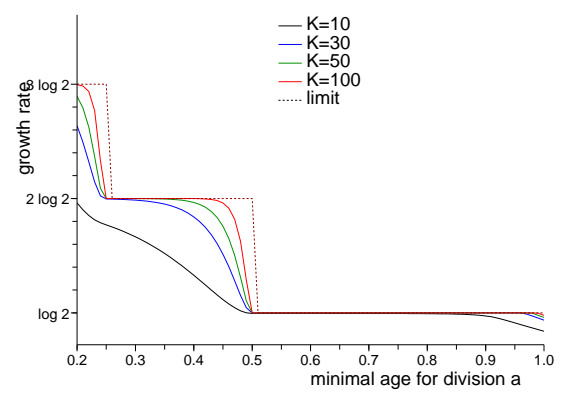

Figure 8. Convergence of the Floquet eigenvalue, when $\tau=1 / 2$. The curves $a \mapsto \lambda(a)$ are shown for $\kappa=10,30,50,100$. The limit $\lambda^{\infty}(a)$ is a staircase function.

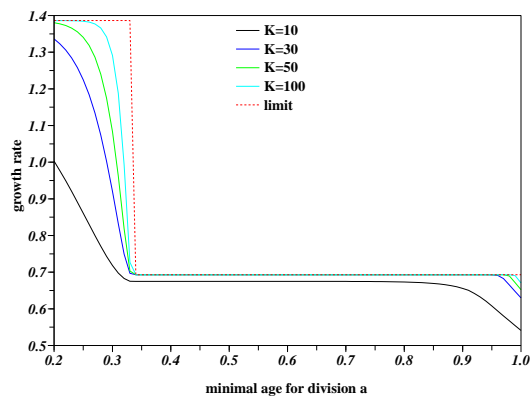

Figure 9. Convergence when $\tau=1 / 3$. Same conventions as in Figure 8.

\section{Appendix A. Appendix: Existence theory for the Floquet eigenvalue}

We shall use the following notation:

- $\tau_{h} B=x \mapsto B(x+h)$, extended to take the value zero on $[0, h]$ if $h<0$,

- $L_{\text {per }}^{\infty}(0, T, X)$ is the space of bounded $T$-periodic functions taking values in $X$, similarly, $C_{\text {per }}(0, T, X)$ is the space of $T$-periodic continuous (with respect to the time variable) functions taking values in $X$. 
Theorem A.1. Assume that $a>0, \kappa>0$, that $\psi$ is nonnegative, bounded, not identically zero and that $B$ positive, bounded, satisfying

$$
\forall t, \quad \int_{0}^{\infty} \psi(t-x) B(x) d x=\int_{0}^{\infty} \psi(t+x) B(x) d x=+\infty .
$$

Then, there exists a unique $\lambda_{F}>0$ such that there exists $(N, \phi)$ in $L_{p e r}^{\infty}\left(0, T, L^{1}\left(\mathbb{R}^{+}\right)\right) \times$ $C_{\text {per }}\left(0, T, L^{\infty}\left(\mathbb{R}^{+}\right)\right)$satisfying (3-4-5). Furthermore, $\phi$ is unique.

Before starting the proof, we give a few remarks on the hypotheses:

- we do not need regularity assumptions on the function $\psi$,

- when $\min (\psi)>0$, the condition (38) is equivalent to $\int_{0}^{\infty} B=+\infty$, in the case $\min (\psi)=0$ it is for instance satisfied if $\min (B)>0$ or at least $\liminf _{+\infty} B>0$, it is not optimal but other conditions would need assumptions on $a$ and $\kappa$. It could be replaced for instance by

$$
\forall t, \quad \int_{a}^{\infty} \kappa \psi(t-x) B(x) d x, \int_{a}^{\infty} \kappa \psi(t+x) B(x) d x>\log 2,
$$

but, as we are studying asymptotic properties, we rather restrict the study to a case where existence does not depend on $a$ nor on $\kappa$,

The proof of Theorem A.1 is based on the method of characteristics and on the Krein-Rutman Theorem, as in [17], but we need more precisions in order to relax the regularity assumptions on $\psi$. For the sake of simplicity we take $\kappa=1$. We set $P(t):=\int_{a}^{\infty} B(x) N(t, x) d x$. Using the methods of characteristics, we have the following integral equations

$$
\begin{aligned}
N(t, 0) & =2 \int_{a}^{\infty} \psi(t) B(x) N(t-x, 0) \exp \left(-\lambda x-\int_{a}^{x} \psi(t-x+s) B(s) d s\right) d x \\
& =: \quad \mathcal{L}_{1}^{\lambda}(N(\cdot, 0))(t), \\
P(t) & =2 \int_{a}^{\infty} \psi(t-x) B(x) P(t-x) \exp \left(-\lambda x-\int_{a}^{x} \psi(t-x+s) B(s) d s\right) d x \\
& =: \quad \mathcal{L}_{2}^{\lambda}(P)(t), \\
\phi(t, 0) & =2 \int_{a}^{\infty} \psi(t+x) B(x) \phi(t+x, 0) \exp \left(-\lambda x-\int_{a}^{x} \psi(t+s) B(s) d s\right) d x \\
& =: \mathcal{L}_{3}^{\lambda}(\phi(\cdot, 0))(t) .
\end{aligned}
$$

We have defined three linear operators on $L_{p e r}^{\infty}(0, T)$. These are well defined as soon as $\lambda>0$. Moreover, we can see $\mathcal{L}_{2}^{\lambda}$ and $\mathcal{L}_{3}^{\lambda}$ as operators on the space $C_{\text {per }}(0, T)$ of T-periodic continuous functions. We have

Lemma A.2. Under the assumptions of Theorem $A .1$, for any $\lambda>0, \mathcal{L}_{2}^{\lambda}$ and $\mathcal{L}_{3}^{\lambda}$ are nonnegative, compact linear operators on $C_{\text {per }}(0, T)$.

Proof. The non negativity and linearity are obvious. We next show the compactness. If we fix $\lambda, a>0$ then for $f$ continuous and $T$-periodic with $\|f\| \leqslant 1$, if we 
write $g=\mathcal{L}_{3}^{\lambda}(f)$, we have

$$
\begin{aligned}
g(t & +h)=2 \int_{a+h}^{\infty} \psi(t+x) B(x-h) f(t+x) \exp \left(\lambda(h-x)-\int_{a+h}^{x} \psi(t+s) B(s) d s\right) d x \\
= & 2 \int_{a}^{\infty} \psi(t+x) B(x-h) f(t+x) \exp \left(\lambda(h-x)-\int_{a+h}^{x} \psi(t+s) B(s) d s\right) d x \\
& -2 \int_{a}^{a+h} \psi(t+x) B(x-h) f(t+x) \exp \left(\lambda(h-x)-\int_{a+h}^{x} \psi(t+s) B(s) d s\right) d x .
\end{aligned}
$$

This leads to

$$
\begin{gathered}
g(t+h)-g(t)=-2 \int_{a}^{a+h} \psi(t+x) B(x-h) f(t+x) \exp \left(\lambda(h-x)-\int_{a+h}^{x} \psi(t+s) B(s-h) d s\right) d x \\
+2\left(e^{\lambda h}-1\right) \int_{a}^{\infty} \psi(t+x) B(x-h) f(t+x) \exp \left(-\lambda x-\int_{a+h}^{x} \psi(t+s) B(s-h) d s\right) d x \\
+2 \int_{a}^{\infty} \psi(t+x)(B(x-h)-B(x)) f(t+x) \exp \left(-\lambda x-\int_{a+h}^{x} \psi(t+s) B(s-h) d s\right) d x \\
+2 \int_{a}^{\infty} \psi(t+x) B(x) f(t+x) e^{-\lambda x}\left(e^{-\int_{a+h}^{x} \psi(t+s) B(s-h) d s}-e^{-\int_{a}^{x} \psi(t+s) B(s) d s}\right) d x \\
=I_{1}+I_{2}+I_{3}+I_{4} .
\end{gathered}
$$

We deal with each $I_{i}$ separately,

$$
\begin{gathered}
\left|I_{1}\right| \leqslant 2\|\psi\|\|B\| e^{\lambda h} h, \\
\left|I_{2}\right| \leqslant 2\|\psi\|\|B\|\left(e^{\lambda h}-1\right) \frac{e^{-\lambda a}}{\lambda},
\end{gathered}
$$

to make $I_{3}$ small, we make the following remark: for any $R>a$,

$$
\left|I_{3}\right| \leqslant 2\|\psi\|\left\|\tau_{h} B-B\right\|_{L^{1}([a, R])}+4\|\psi\|\|B\| \int_{R}^{\infty} e^{-\lambda x} d x .
$$

Observe that for all $a$ and $R, \lim _{h \rightarrow 0^{+}}\left\|\tau_{h} B-B\right\|_{L^{1}([a, R])}=0$ (indeed, this is obvious if $B$ is continuous, and, by density of continuous functions in $L^{1}([a, R])$, the same is true under the present assumptions). Since $\lambda$ is positive, the second term in (41) can be made arbitrarily small by choosing $R$ large enough, and then, the first term can be made arbitrarily small by choosing $h$ small enough. It follows that for all $\epsilon>0$, we have

$$
\left|I_{3}\right| \leqslant \varepsilon \quad \text { for } h \text { small enough. }
$$

The same method can be applied to $I_{4}$. Finally we have the equicontinuity of $\mathcal{L}_{3}^{\lambda}(B)$ where $B$ is the unit ball for the supremum norm. Thanks to Arzela-Ascoli theorem, $\mathcal{L}_{3}^{\lambda}$ is compact.

We are now in position to apply Krein-Rutman theorem, there exist $U_{2}, U_{3}$ nonnegative eigenvectors of $\mathcal{L}_{2}^{\lambda}, \mathcal{L}_{3}^{\lambda}$ associated to their respective spectral radii $\rho_{2}(\lambda), \rho_{3}(\lambda)$. We have now

\section{Lemma A.3.}

$$
\begin{gathered}
\rho_{i}(\lambda), U_{i}>0, \\
\mathcal{L}_{1}^{\lambda}\left(\psi U_{2}\right)=\rho_{2}(\lambda) \psi U_{2}, \\
\rho_{3}=\rho_{2} .
\end{gathered}
$$


Proof. The second statement is a straightforward computation, we prove the first by contradiction: if $U_{2}$ vanishes, then, for some $t$,

$$
2 \int_{a}^{\infty} \psi(t-x) B(x) U_{2}(t-x) \exp \left(-\lambda x-\int_{a}^{x} \psi(t-x+s) B(s) d s\right) d x=0,
$$

as $B>0$, it would mean thanks to $T$-periodicity $\psi B \equiv 0$ which would lead to $U_{2} \equiv 0$. Finally, as $U_{2}$ does not vanish then $\mathcal{L}_{2}^{\lambda}\left(U_{2}\right)>0$ and therefore, $\rho_{2}(\lambda)>0$. The equality comes from the duality of operators $\mathcal{L}_{1}$ and $\mathcal{L}_{3}$, we have

$$
\begin{aligned}
\rho_{2} \int_{0}^{T} \psi(t) U_{2}(t) U_{3}(t) d t & =\int_{0}^{T} \mathcal{L}_{1}\left(\psi U_{2}\right) \cdot U_{3}(t) d t=\int_{0}^{T} \psi U_{2} \mathcal{L}_{3}\left(U_{3}\right) d t \\
& =\rho_{3} \int_{0}^{T} \psi(t) U_{2}(t) U_{3}(t) d t
\end{aligned}
$$

therefore $\rho_{2}=\rho_{3}=\rho$.

To end the proof, we need to find $\lambda$ such that $\rho(\lambda)=1$. Obviously, $\rho$ is a decreasing function that vanishes at infinity. Since

$2 \int_{a}^{\infty} \psi(t+x) B(x) \exp \left(-\int_{a}^{x} \psi(t+s) B(s) d s\right) d x=2\left[\exp \left(-\int_{a}^{x} \psi(t+s) B(s) d s\right)\right]_{a}^{\infty}$

$$
=2 \text {, }
$$

$\rho \rightarrow 2$ as $\lambda \rightarrow 0$. Therefore there exists a unique $\lambda$ satisfying $\rho(\lambda)=1$. Up to a renormalization, $\phi$ and $P$ are unique, and therefore so is $N$. This ends the proof of Theorem A.1.

\section{ACKNOWLEDGEMENT}

The authors thank Jean Clairambault and Benoit Perthame for numerous insightful remarks and discussions. Most of this work has been achieved during the $\mathrm{PhD}$ of the second author at Laboratoire Jacques Louis Lions (Paris) and INRIA Rocquencourt.

\section{REFERENCES}

[1] M. Akian, R. Bapat, and S. Gaubert. Asymptotics of the Perron eigenvalue and eigenvector using max algebra. C. R. Acad. Sci. Paris., 327, Série I:927-932, 1998.

[2] N. Bacaer and X. Abdurahman. Resonance of the epidemic threshold in a periodic environment. Journal of Mathematical Biology, 57(5):649-673, November 2008.

[3] J. Clairambault, S. Gaubert, and T. Lepoutre. Comparison of Perron and Floquet eigenvalues in age structured cell division cycle models. Math. Model. Nat. Phenom., 4(3):183-209, 2009. Also eprint arXiv:0812.0803.

[4] J. Clairambault, S. Gaubert, and T. Lepoutre. Circadian rhythm and cell population growth. Mathematical and Computer Modelling, 53(7-8):1558 - 1567, 2011. Mathematical Methods and Modelling of Biophysical Phenomena.

[5] J. Clairambault, S. Gaubert, and B. Perthame. An inequality for the Perron and Floquet eigenvalues of monotone differential systems and age structured equations. C. R. Math. Acad. Sci. Paris, 345(10):549-554, 2007.

[6] J. Clairambault, P. Michel, and B. Perthame. Circadian rhythm and tumour growth. C. R. Acad. Sci., 342(1):17-22, 2006.

[7] J. Clairambault, P. Michel, and B. Perthame. A mathematical model of the cell cycle and its circadian control. In D. I.A., B. L., B. H., de Vries G., and H. H. P., editors, Mathematical modeling of Biological Systems, pages 247-259. Birkhäuser, 2007.

[8] B. F. Dibrov, A. M. Zhabotinsky, Y. A. Neyfakh, M. P. Orlova, and L. I. Churikova. Mathematical model of cancer chemotherapy. Periodic schedules of phase-specific cytotoxic-agent administration increasing the selectivity of therapy. Math. Biosci., 73(1):1-31, 1985. 
[9] O. Diekmann, H. J. A. M. Heijmans, and H. R. Thieme. On the stability of the cell-size distribution. II. Time-periodic developmental rates. Comput. Math. Appl. Part A, 12(4-5):491-512, 1986. Hyperbolic partial differential equations, III.

[10] M. Doumic. Analysis of a population model structured by the cells molecular content. Mathematical Modelling of Natural Phenomena, 2(3):121-152, 2007.

[11] A. Goldbeter. Biochemical oscillations and cellular rhythms. Cambridge University Press, 1997.

[12] W. O. Kermack and A. McKendrick. A Contribution to the Mathematical Theory of Epidemics. Proceedings of the Royal Society of London. Series A, Containing Papers of a Mathematical and Physical Character, 115(772):700-721, Aug. 1927.

[13] F. Levi and U. Schibler. Circadian rhythms: mechanisms and therapeutic implications. Annu Rev Pharmacol Toxicol, 47:593-628, 2007.

[14] P. Magal. Compact attractors for time-periodic age-structured population models. Electron. J. Differential Equations, pages No. 65, 35 pp. (electronic), 2001.

[15] A. Marshall and I. Olkin. Inequalities: Theory of majorization and its applications. Academic Press, New York, 1979.

[16] J. Metz and O. Diekmann. The dynamics of physiologically structured populations, volume 68 of L.N. in biomathematics. Springer, 1986.

[17] P. Michel, S. Mischler, and B. Perthame. General relative entropy inequality: an illustration on growth models. J. Math. Pures et Appl., 84(9):1235-1260, May 112005.

[18] B. Perthame. Transport equations in biology. Birkhäuser, 2007.

[19] H. R. Thieme. Renewal theorems for linear periodic Volterra integral equations. J. Integral Equations, 7(3):253-277, 1984.

[20] P. Thieullen and E. Garibaldi. Description of some ground states by puiseux techniques. J. Stat. Phys., 146:125-180, 2012.

[21] G. F. Webb. Theory of nonlinear age-dependent population dynamics, volume 89 of Monographs and Textbooks in Pure and Applied Mathematics. Marcel Dekker Inc., New York, 1985.

E-mail address: stephane.gaubert@inria.fr

E-mail address: thomas.lepoutre@inria.fr 